\title{
La mortalité chez les adultes autochtones vivant en milieu urbain au Canada, 1991-2001*
}

\author{
M. Tjepkema, M.H.P. (1); R. Wilkins, M. Urb. (1,2); S. Senécal, Ph. D. (3,4); É. Guimond, Ph. D. (3,4); C. Penney, M.A. (3)
}

${ }^{*}$ Cet article fait l'objet d'une publication conjointe par Preventing Chronic Disease et Maladies chroniques au Canada. Maladies chroniques au Canada en est le premier éditeur, Preventing Chronic Disease le second.

\section{Résumé}

Objectif : Comparer les profils de mortalité des adultes autochtones et non autochtones vivant en milieu urbain.

Méthodologie : En s’appuyant sur l'Étude canadienne de suivi de la mortalité selon le recensement 1991-2001, notre étude retrace l'évolution de la mortalité jusqu'au 31 décembre 2001 au sein d'un échantillon de $15 \%$ des adultes canadiens, constitué de 16300 personnes autochtones et 2062700 non autochtones vivant dans des zones urbaines au 4 juin 1991. Étant donné que le recensement de 1991 n’a pas recueilli de renseignements sur l'identité autochtone, l'appartenance à la population autochtone a été définie selon l'origine ethnique (l'ascendance), le statut d'Indien inscrit ou l'appartenance à une bande indienne ou à une Première nation.

Résultats : L'espérance de vie à l'âge de 25 ans des hommes et des femmes autochtones vivant en milieu urbain était, par rapport à celle des hommes et des femmes non autochtones vivant en milieu urbain, respectivement plus courte de 4,7 années et 6,5 années. Les rapports des taux de mortalité des hommes et des femmes autochtones étaient particulièrement élevés lorsqu'il s'agissait de décès liés à la consommation d'alcool, d'accidents de la route et de maladies infectieuses, dont le VIH/sida. Pour la plupart des causes de décès, les taux de mortalité étaient plus élevés chez les adultes autochtones que chez les non autochtones. Le statut socioéconomique explique en grande partie ces écarts.

Conclusion : Les résultats de cette étude contribuent à combler une lacune dans la connaissance de la mortalité des populations autochtones vivant en milieu urbain au Canada.

Mots-clés : Peuple autochtone, Premières nations, Métis, Inuit, Indiens d'Amérique du Nord, taux de mortalité, taux de mortalité normalisés selon l'âge, espérance de vie

\section{Introduction}

Le nombre d'Autochtones (membres des Premières nations, Métis et Inuits) vivant en milieu urbain au Canada a considérablement augmenté au cours du dernier demi-siècle; en 1950, environ $7 \%$ d'entre eux vivaient dans les régions urbaines du Canada ${ }^{1}$, contre $54 \%$ en $2006^{2}$.
Cependant, l'ampleur de la recherche sur la santé de la population autochtone vivant en milieu urbain n'est pas proportionnelle à cette croissance au sein de la population totale ${ }^{3,4}$, pas plus qu'elle ne reflète cette proportion croissante au sein des populations autochtones.
Les Autochtones choisissent de vivre en milieu urbain pour différentes raisons : raisons familiales, perspectives d'emploi, éducation, formation et santé (par exemple, proximité des services médicaux ${ }^{5,6}$ ). Les défis auxquels ils font face sont différents de ceux de leurs homologues ruraux, entre autres trouver un logement convenable et localiser les services et le soutien disponibles pour les aider à mieux vivre cette transition ${ }^{5,7}$.

Même s'il est de notoriété publique que, comparativement aux autres Canadiens, les Autochtones supportent un fardeau disproportionné de mortalité et de maladie ${ }^{8-12}$, les données concernant ceux qui résident en milieu urbain sont moins bien connues ${ }^{13}$. De même, alors que l'espérance de vie des membres des Premières nations, des Métis et des Inuits est considérablement plus courte que celle de la population générale ${ }^{14-18}$, il est, malgré tout, difficile d'évaluer les indicateurs de la mortalité des Autochtones vivant en milieu urbain au Canada, parce que les identificateurs autochtones ne sont pas consignés lors de l'enregistrement des décès dans la plupart des provinces. Les courbes de mortalité des Indiens inscrits vivant au Manitoba et en Colombie-Britannique ont été analysées et elles ont permis d'obtenir des résultats à l'échelle infraprovinciale, dont les villes de Winnipeg ${ }^{19}$ et de Vancouver ${ }^{20}$. Toutefois, ces études n'éclairent qu'une partie du tableau, car elles ne tiennent pas compte des membres des Premières nations qui ne sont pas inscrits aux termes de la Loi sur les Indiens, ni des Métis et des Inuits, et elles ne fournissent aucun renseignement sur les Autochtones habitant dans les autres zones urbaines canadiennes.

Rattachement

1 Division de l'analyse de la santé, Statistique Canada, Ottawa (Ontario)

2 Département d'épidémiologie et de médecine sociale, Université d'Ottawa, Ottawa (Ontario)

3 Direction de la recherche stratégique et de l'analyse, Affaires indiennes et du Nord Canada, Gatineau (Québec)

4 Département de sociologie, Université Western Ontario, London (Ontario)

Correspondance : Michael Tjepkema, Division de l'analyse de la santé de Statistique Canada, Immeuble R.H. Coats, 24étage, 100, allée Tunney’s Pasture, Ottawa (Ontario) K1A 0T6; tél. : 613-951-3896; téléc. : 613-951-3959; courriel : michael.tjepkema@statcan.gc.ca 
L'Étude canadienne de suivi de la mortalité selon le recensement 1991-2001 offre l'occasion d'étudier les profils de mortalité d'un nombre suffisamment important d'Autochtones vivant en zone urbaine au début de la période de suivi, dans toutes les provinces et territoires, et qu'ils soient ou non inscrits aux termes de la Loi sur les Indiens.

Les objectifs de cet article sont les suivants : 1) déterminer dans quelle mesure les Autochtones adultes vivant en milieu urbain courent le risquent de mourir prématurément; 2) mesurer l'espérance de vie et les probabilités de survie jusqu'à l'âge de 75 ans et 3 ) déterminer les causes comportant le risque le plus élevé de décès.

\section{Méthodologie}

\section{Sources des données}

L'Étude canadienne de suivi de la mortalité selon le recensement a été menée auprès d'un échantillon de 15 \% ( $\mathrm{n}=2735$ 152) de la population canadienne âgée de 25 ans ou plus, à l'exclusion des pensionnaires d'établissements institutionnels, et dont les répondants avaient tous rempli le questionnaire détaillé du recensement de 1991. Un suivi de la mortalité a été effectué auprès de cette cohorte du 4 juin 1991 au 31 décembre 2001. La création de la base des données de l'étude de suivi de la mortalité selon le recensement a exigé deux mises en relation, parce que les fichiers électroniques des données du recensement ne contenaient pas de noms, mais qu'il fallait disposer des noms pour trouver les décès correspondants. Au moyen de variables communes telles que la date de naissance, le code postal, ainsi que la date de naissance du conjoint ou de la conjointe (s'il y avait lieu), on a d'abord procédé à un couplage probabiliste du fichier du recensement avec un fichier nominatif codé extrait des données non financières des personnes ayant fait une déclaration de revenu. Le fichier de recensement et le fichier nominatif codé ont ensuite été jumelés à la Base canadienne de données sur la mortalité en utilisant des méthodes de couplage d'enregistrements probabilistes ${ }^{21}$ - une approche semblable à celle qui avait servi dans le cadre d'autres études de suivi de la mortalité effectuées par Statistique Canada $^{22}$. Les détails complets concernant la réalisation et le contenu de ce fichier couplé sont présentés ailleurs ${ }^{16}$.

\section{Admissibilité}

Seuls les citoyens ayant rempli le questionnaire détaillé lors du recensement de 1991, âgés de 25 ans ou plus le jour du recensement et résidents canadiens, pouvaient faire partie de cette cohorte. Les rapports sur la qualité des données estiment que 3,4 \% des résidents canadiens de tous âges n’ont pas été recensés en 1991. Selon toute probabilité, bon nombre des personnes non recensées étaient plus jeunes, plus mobiles, à plus faible revenu, plus fréquemment d'ascendance autochtone $^{23,24}$ ou sans abri. Seuls les membres de la cohorte qui vivaient dans des régions urbaines (voir la section Définitions) le jour du recensement étaient ciblés par cette étude. Le questionnaire détaillé du recensement est généralement distribué à $20 \%$ des ménages canadiens, à tous les résidents des réserves indiennes, à tous les résidents de bon nombre de collectivités éloignées et du Nord et à tous les résidents de logements collectifs non institutionnels. Comme il a été, de plus, nécessaire d'obtenir les noms codés provenant des données sur les personnes ayant effectué une déclaration de revenus, seules ces personnes pouvaient faire l'objet de l'étude de suivi de la mortalité. Il n'y avait toutefois pas de différences significatives liées aux caractéristiques démographiques et socioéconomiques entre les répondants du recensement admissibles à l'étude et ceux qu'on a pu jumeler à un fichier nominatif (tableaux en annexe A, B et C).

\section{Techniques d'analyse}

Pour chaque membre de la cohorte, les jours-personnes de suivi ont été calculés pour la période allant du début de l'étude (le 4 juin 1991) jusqu'au décès, la date d'émigration (déterminée à partir du fichier nominatif et connue pour 1991 seulement) ou encore jusqu'à la fin de l'étude (le 31 décembre 2001). Les années-personnes à risque ont été calculées en divisant les jours-personnes de suivi par 365,25.
En utilisant la structure démographique des Autochtones de la cohorte ${ }^{1}$ (annéespersonnes à risque) dans son ensemble et en la considérant comme population type, nous avons calculé les taux de mortalité selon l'âge et le sexe par groupe d'âge de cinq ans (à la date de référence) puis les taux de mortalité normalisés selon l'âge (TMNA) pour des sous-groupes de la population. Nous avons calculé des intervalles de confiance (IC) à $95 \%$ correspondant aux TMNA selon la façon décrite par Carrière et $\operatorname{Roos}^{25}$. Une méthode semblable a permis de calculer les IC pour les rapports de taux (RT) des TMNA.

Pour les analyses selon l'âge, les membres de la cohorte ont été répartis en groupes d'âge de 10 ans, de 25-34 ans à 65-74 ans, en plus de la catégorie des 75 ans et plus. La plupart des analyses ont utilisé l'âge à la date de référence (le 4 juin 1991), les analyses des tables de survie étant fondées, elles, sur l'âge au début de chaque année de suivi.

Les tables de survie par période et par sexe, de même que les erreurs types et les IC à $95 \%$ correspondants ont été calculés d'après la méthode de Chiang ${ }^{26}$. Nous avons d'abord converti l'âge à la date de référence en âge au début de chaque année de suivi, puis nous avons calculé séparément les décès et les années-personnes à risque pour chaque année (ou année partielle) de suivi. Les décès et les années-personnes à risque ont par la suite été regroupés selon l'âge au début de chaque année de suivi, avant le calcul des tables de survie.

Nous avons calculé, en suivant le modèle de Cox, les rapports de risques proportionnels de mortalité selon le sexe, en contrôlant d'abord l'effet de l'âge (nombre d'années), puis le lieu de résidence (régions métropolitaines, petits centres urbains), le statut parental (monoparentalité - oui ou non), le degré de scolarité (sans diplôme d'études secondaires, diplôme d'études secondaires, diplôme d'études postsecondaires, diplôme d'études universitaires), le quintile de revenu (1 à 5), le niveau de compétence professionnelle (poste professionnel, poste de gestion, poste spécialisé,

† Toute personne ayant déclaré dans le questionnaire détaillé du recensement une ascendance d'Indien d'Amérique du Nord, de Métis ou d'Inuit, un statut d'Indien inscrit ou l'appartenance à une bande indienne de l'Amérique du Nord ou à une Première nation - voir la section Définitions. 
technique ou de supervision, poste de spécialisation moyenne et poste non spécialisé, sans profession), la situation relative à l'emploi (en emploi, au chômage, inactif) et le lieu de naissance (au Canada ou ailleurs). Le lieu de naissance a été inclus dans ces modèles afin de réduire l'" effet immigrants en bonne santé » parmi les membres non autochtones de la cohorte. Les définitions détaillées de ces variables (qui ont toutes été établies et contrôlées seulement à la date de référence) ont été fournies précédemment ${ }^{16}$. Nous avons interprété les écarts en matière de surmortalité entre le modèle corrigé selon l'âge et le modèle pleinement corrigé comme un effet des variables socioéconomiques (lieu de résidence, statut parental, degré de scolarité, etc.) sur l'importance des disparités entre les adultes autochtones et non autochtones vivant en milieu urbain. La proportion de mortalité excédentaire attribuée aux variables socioéconomiques a été calculée de la manière suivante : différence entre les rapports ajustés par âge et les rapports pleinement ajustés pour les Autochtones (oui/non) divisée par le rapport ajusté par âge moins 1 .

La cause de décès pour chaque personne décédée au cours de la période 1991-1999 a été codée selon la Classification internationale des maladies, neuvième révision de l'Organisation mondiale de la Santé $\left(\right.$ CIM-9) ${ }^{27}$ et les causes de décès en 2000 ou 2001 selon la Dixième révision (CIM-10) ${ }^{28}$. Pour les analyses selon les causes de décès, les décès ont été regroupés en fonction des sections de la CIM-9, des catégories présentées dans les différentes sections, et des facteurs de risque (liés au tabagisme, à l'alcool, aux drogues ou susceptibles d'être évités par intervention médicale ${ }^{29,30}$. Ces données sont présentées dans le tableau D en annexe.

\section{Définitions}

Le recensement de 1991 n'a pas recueilli de données concernant l'autoidentification avec un groupe autochtone (Indiens de l'Amérique du Nord, Métis ou Inuit). Pour les besoins de l'analyse, nous avons défini cette population au moyen de deux questions, faisant ressortir trois des caractéristiques propres à l'identité autochtone :
1. Ascendance : à la question $n^{\circ} 15$ du questionnaire détaillé, on demandait à chaque répondant d'indiquer à quel(s) groupe(s) ethnique(s) ou culturel(s) ses ancêtres appartenaient, à partir d'une liste de 15 choix (dont Indien d'Amérique du Nord, Métis et Inuit) ${ }^{31}$. On demandait aux répondants de préciser toutes les origines ethniques qui s'appliquaient.

2. Statut d'Indien inscrit : à la question $\mathrm{n}^{\circ} 16$ du questionnaire détaillé, on demandait " le répondant est-il un Indien inscrit aux termes de la Loi sur les Indiens du Canada?» (oui ou non).

3. Appartenance à une bande indienne ou à l'une des Premières nations : à la question $\mathrm{n}^{\circ} 16$, on demandait au répondant, dans le cas où il appartenait à une bande indienne ou à l'une des Premières nations, de préciser dans la case prévue à cet effet de quelle bande ou nation il s'agissait.

Dans le cadre de cette étude, une personne était considérée comme Autochtone si elle déclarait une ascendance autochtone à l'exclusion de toute autre ascendance, ou deux ascendances autochtones ou plus (avec ou sans autres ascendances), ou si elle déclarait être Indien inscrit ou appartenir à une bande indienne ou à l'une des Premières nations. D’après une analyse des données du recensement de 1996 présentant les recoupements entre les origines ethniques en lien avec l'identité autochtone ${ }^{32}$, plus de $94 \%$ des participants au recensement de 1996 répondant à nos critères fondés sur l'ascendance se percevaient comme Autochtones. Le nombre d'Autochtones (particulièrement de Métis) est sous-estimé dans le cadre de cette étude, parce que les répondants comptant une seule ascendance autochtone mais au moins une ascendance non-autochtone parmi leurs ancêtres sont considérés comme non-Autochtones (sauf si ils déclarent être Indien inscrit ou appartenir à une bande indienne ou à l'une des Premières nations).

La " région urbaine » peut être définie différemment selon l'objet de la recherche et les données dont on dispose ${ }^{33}$. Notre définition se distingue de la définition normalisée du mot "urbain $»^{31}$ utilisée dans le cadre du recensement : nous utilisons l'expression "région urbaine " pour désigner les régions métropolitaines de recensement (désignant une collectivité de 100000 habitants et plus) et les agglomérations de recensement (collectivité de 10000 habitants et plus), à l'exclusion des réserves et des autres établissements autochtones faisant partie de ces zones. Nous n'avons pas considéré les autres zones urbaines dans le cadre de cette étude.

\section{Membres de la cohorte, taux de couplage, décès et années-personnes à risque}

Le tableau en annexe A indique qu'il y avait 2,6 millions de répondants au recensement vivant dans les régions urbaines canadiennes admissibles à l'étude, dont 25500 Autochtones adultes. Les taux de couplage avec le fichier nominatif (couplage reliant membres de la cohorte et répondants au questionnaire détaillé) des Autochtones vivant en milieu urbain (61 \% des hommes et $66 \%$ des femmes) étaient inférieurs à ceux des non-Autochtones (80\% des hommes et $76 \%$ des femmes). En dépit de ce taux de couplage plus faible, les caractéristiques démographiques et socioéconomiques des membres autochtones de la cohorte vivant en milieu urbain étaient, en général, semblables à celles de l'ensemble de la population autochtone adulte admissible, à l'exclusion des cas suivants : les personnes qui avaient un emploi, celles qui avaient un revenu plus élevé et celles qui étaient mariées se révélèrent légèrement plus susceptibles d'obtenir un couplage réussi, un résultat semblable à celui obtenu par les membres non autochtones de la cohorte, ce qui semble indiquer que notre échantillon d'Autochtones vivant en milieu urbain n'était pas biaisé en ce qui concerne ces caractéristiques (tableaux en annexe B et C).

L'examen des décès survenus en 1991, qui pouvaient être compilés indépendamment en utilisant la Base canadienne de données sur la mortalité ou le fichier nominatif, a permis d'estimer leur confirmation dans approximativement $97 \%$ des cas dans la cohorte étudiée pour le suivi de mortalité (1991-2001), et $95 \%$ à $96 \%$ pour la population autochtone. 
Au total, la cohorte suivie pour la mortalité comprenait 16300 adultes autochtones vivant en milieu urbain, totalisant 166570 annéespersonnes à risque et 1126 décès survenus au cours de la période de suivi de 11 ans (tableau en annexe A).

\section{Résultats}

Selon le recensement de 1991, on estime à 259800 le nombre d'Autochtones âgés de 25 ans ou plus, ce qui représente 1,5\% de la population adulte totale du Canada. Environ 45 \% d'entre eux vivaient dans des régions urbaines (30 \% dans des régions métropolitaines et $15 \%$ dans de petits centres urbains). En comparaison, $78 \%$ des personnes non autochtones vivaient dans des régions urbaines (62\% dans des régions métropolitaines et $16 \%$ dans de petits centres urbains). Dans l'ensemble des régions urbaines, $69 \%$ des Autochtones faisaient partie des Premières nations (40 \% d'Indiens inscrits, $29 \%$ d'Indiens non inscrits), $28 \%$ étaient métis et $3 \%$ inuits.

$\mathrm{Au}$ début de la période de suivi (le 4 juin 1991), on a comptabilisé dans la cohorte vivant dans une région métropolitaine ou dans un petit centre urbain 16300 Autochtones. Le tableau 1 illustre les caractéristiques démographiques et socioéconomiques des membres autochtones et non autochtones de la cohorte vivant en milieu urbain au Canada. Presque les trois quarts des Autochtones membres de la cohorte étaient âgés de 25 à 44 ans, contre $54 \%$ des adultes non autochtones du même groupe d'âge. Environ $44 \%$ des adultes autochtones n'avaient pas terminé leurs études secondaires (contre $31 \%$ des adultes non autochtones) et $61 \%$ font partie des deux quintiles de revenus les plus faibles (contre $36 \%$ des adultes non autochtones).

\section{Espérance de vie à 25 ans et probabilité de survie jusqu'à 75 ans}

L'espérance de vie à 25 ans (qui présuppose d'avoir survécu jusqu'à cet âge) des adultes autochtones des deux sexes vivant en milieu urbain était sensiblement plus courte que celle des adultes non autochtones vivant en milieu urbain. Le tableau 2 indique que l'espérance de vie à 25 ans des hommes autochtones vivant en milieu urbain était de 48,1 années (IC à $95 \%$ : 47,1 à 49,1 ), comparativement à 52,8 années (IC à $95 \%: 52,8$ à 52,9 ) pour les hommes non autochtones vivant en milieu urbain, soit une différence de 4,7 années. L'espérance de vie à l'âge de 25 ans des femmes autochtones vivant en milieu urbain était plus longue que celle des hommes autochtones dans la même situation, mais l'écart entre l'espérance de vie des femmes autochtones vivant en milieu urbain (52,7 années; IC à $95 \%$ : 51,7 à 53,7) et celle des femmes non autochtones dans la même situation (59,2 années; IC à $95 \%: 59,2$ à 59,3) était plus grand (6,5 années). L'espérance de vie des Autochtones adultes vivant dans des régions métropolitaines était semblable à celle des Autochtones adultes résidant dans de petits centres urbains.

Le tableau 2 illustre la probabilité de survie jusqu'à l'âge de 75 ans, présupposant la survie jusqu'à l'âge de 25 ans, des membres de la cohorte vivant en milieu urbain. Environ $52 \%$ (IC à $95 \%: 48$ à 56) des hommes autochtones vivant en milieu urbain pouvaient espérer vivre jusqu'à l'âge de 75 ans comparativement à $65 \%$ (IC à $95 \%$ : 64 à 65) des hommes non autochtones vivant en milieu urbain, soit un écart de 12 points. En ce qui concerne les femmes autochtones vivant en milieu urbain, $63 \%$ (IC à $95 \%$ : 59 à 66) pouvaient espérer vivre jusqu'à l'âge de 75 ans, comparativement à $80 \%$ (IC à $95 \%$ : 79 à 80) des femmes non autochtones vivant en milieu urbain, soit un écart de 17 points.

\section{Taux de mortalité selon l'âge et taux de mortalité normalisés selon l'âgé}

Le tableau 3 illustre les rapports de taux (RT) de mortalité selon l'âge et les RT normalisés selon l'âge pour les adultes autochtones et non autochtones vivant en milieu urbain. Dans l'ensemble, les rapports de taux se révélèrent sensiblement plus élevés dans le cas des hommes autochtones $(\mathrm{RT}=1,56$; IC à $95 \%: 1,43$ à 1,70$)$ et des femmes autochtones (RT $=1,94$; IC à $95 \%: 1,78$ à 2,11) que chez les non autochtones. Pour les deux sexes, les rapports de taux atteignaient des pourcentages plus élevés parmi les plus jeunes, et diminuaient à mesure que les gens vieillissaient.

\section{Les causes de décès}

Le tableau 4 montre les taux de mortalité normalisés selon l'âge (TMNA) répartis selon les principales causes de décès des Autochtones de la cohorte vivant en milieu urbain tandis que le tableau 5 illustre les rapports de TMNA par rapport aux membres non autochtones de la cohorte vivant dans le même contexte urbain. Les causes de décès les plus courantes parmi les hommes autochtones étaient les maladies de l'appareil circulatoire (33\% du total des TMNA), suivies par les différents types de cancer (23\%) et les causes externes (16\%) - un classement semblable à celui des hommes non autochtones vivant en milieu urbain. Dans le cas des femmes autochtones vivant en milieu urbain, les causes de décès les plus courantes étaient également les maladies de l'appareil circulatoire (29 \% du total des TMNA), suivies par les différents types de cancer (26\%), les causes externes (10\%) et les maladies de l'appareil digestif ( $9 \%$ ); chez les femmes non autochtones vivant en milieu urbain, la cause de décès la plus courante était le cancer (42\%), suivi par les maladies de l'appareil circulatoire (29 \%), les maladies de l'appareil respiratoire $(6 \%)$ et les causes externes $(6 \%)$.

Le tableau 5 montre les rapports de taux (RT) normalisés selon l'âge répartis selon les causes principales de décès. (Le nombre de décès correspondants et les TMNA sont indiqués dans le tableau 4 et le tableau $\mathrm{D}$ en annexe.) Les rapports de taux concernant les hommes autochtones vivant en milieu urbain étaient relativement élevés pour les décès causés par des maladies de l'appareil circulatoire (RT = 1,50; IC à $95 \%: 1,29$ à 1,74) comme la cardiopathie ischémique ( $\mathrm{RT}=1,52$; IC à $95 \%: 1,26$ à 1,83), ce qui n’était pas le cas pour tous les types de cancer combinés $(\mathrm{RT}=1,09$; IC à $95 \%: 0,92$ à 1,30); néanmoins, le rapport de taux était élevé pour les décès attribuables à des cancers de la trachée, des bronches ou des poumons $(\mathrm{RT}=1,42$; IC à $95 \%: 1,08$ à 1,88) surtout 
chez les hommes autochtones vivant dans les régions métropolitaines au début de la période de suivi. Les rapports de taux chez les hommes autochtones vivant en milieu urbain étaient particulièrement élevés pour les maladies de l'appareil digestif (RT $=3,00$; IC à $95 \%$ : 2,09 à 4,30), toutes les causes externes $(\mathrm{RT}=2,80$; IC à $95 \%: 2,29$ à 3,43$)$ spécialement les accidents de la route $(\mathrm{RT}=3,51$; IC à $95 \%: 2,32$ à 5,32$)$ et à un degré moindre, les suicides ( $\mathrm{RT}=1,57$; IC à $95 \%: 1,04$ à 2,38) - de même que les décès résultant de maladies infectieuses $(\mathrm{RT}=2,04$; IC à $95 \%: 1,33$ à 3,11$)$, dont le $\mathrm{VIH} /$ sida $(\mathrm{RT}=2,03$; IC à $95 \%: 1,22$ à 3,39). À quelques exceptions près (notamment les maladies de l'appareil endocrinien et le suicide), les rapports de taux des hommes autochtones vivant dans des régions métropolitaines étaient semblables à ceux des hommes autochtones résidant dans de petits centres urbains.

Les rapports de taux concernant les femmes autochtones demeuraient élevés pour presque toutes les causes principales de décès, à l'exception du cancer du sein. Par exemple, les rapports de taux étaient élevés pour ce qui concerne les maladies de l'appareil circulatoire (RT $=1,93$; IC à $95 \%: 1,64$ à 2,28) et tous les types de cancer combinés (RT $=1,21$; IC à $95 \%$ : 1,03 à 1,42), les deux causes de décès les plus courantes. Les rapports de taux étaient particulièrement élevés pour les décès causés par les maladies infectieuses $(\mathrm{RT}=5,76$; IC à $95 \%: 3,68$ à 9,01) dont le $\mathrm{VIH} /$ sida $(\mathrm{RT}=10,65$; IC à $95 \%: 4,56$ à 24,88 ), les maladies de l'appareil digestif $(\mathrm{RT}=4,82$; IC à $95 \%: 3,67$ à 6,34), les causes externes (RT $=3,37$; IC à $95 \%$ : 2,59 à 4,37) - surtout les accidents de la route ( $\mathrm{RT}=4,13$; IC à $95 \%: 2,46$ à 6,93) - et les maladies de l'appareil endocrinien comme le diabète sucré ( $\mathrm{RT}=2,61$; IC à $95 \%: 1,73$ à 3,94). À quelques exceptions près, les rapports de taux obtenus chez les femmes autochtones vivant dans des régions métropolitaines étaient plus élevés que ceux des femmes résidant dans de petits centres urbains.

Dans le tableau 5 , les décès sont répartis en en fonction de différents facteurs : ceux liés au tabagisme, à la consommation d'alcool ou de drogues, ou susceptibles d'être évités par intervention médicale ${ }^{28,29}$. Comparativement aux hommes et aux femmes non autochtones vivant en milieu urbain, les taux concernant les causes liées au tabagisme (qui comptent respectivement pour $15 \%$ et $7 \%$ du total des TMNA chez les hommes et les femmes autochtones vivant en milieu urbain) étaient élevés chez les hommes autochtones $(\mathrm{RT}=1,46$; IC à $95 \%: 1,17$ à 1,82) comme chez les femmes autochtones $(\mathrm{RT}=1,36$; IC à $95 \%: 1,04$ à 1,78 ) vivant en milieu urbain. Les taux concernant les causes liées à la consommation d'alcool étaient considérablement plus élevés chez les hommes autochtones $(\mathrm{RT}=4,55$; IC à $95 \%: 3,14$ à 6,61$)$ et les femmes autochtones (RT $=11,44$; IC à $95 \%: 8,02$ à 16,34), et les taux concernant les décès liés à la consommation de drogues étaient aussi sensiblement plus élevés chez les hommes autochtones $(\mathrm{RT}=3,71$; IC à $95 \%: 2,22$ à 6,22$)$ et les femmes autochtones (RT $=6,43$; IC à $95 \%: 4,26$ à 9,73). Les taux de décès prématurés (avant l'âge de 75 ans) susceptibles d'être évités par intervention médicale (par exemple les décès résultant du cancer du sein ou du col de l'utérus, de maladies infectieuses ou de maladie cérébrovasculaire, ou encore de pneumonie ou de grippe) étaient également nettement plus élevés chez les Autochtones d'âge adulte des deux sexes.

Au sein de la population adulte autochtone vivant en milieu urbain, les hommes étaient plus susceptibles que les femmes de mourir de causes liées au tabagisme (TMNA = 130 par 100000 années-personnes à risque contre 58), mais moins susceptibles de mourir de causes susceptibles d'être évitées par intervention médicale (69 contre 92), une structure similaire à celle de la population non autochtone. Les risques de décès liés à la consommation d'alcool étaient légèrement plus élevés chez les hommes autochtones vivant en milieu urbain que chez les femmes autochtones vivant dans le même contexte (42 contre 34), à la différence de la population non autochtone, au sein de laquelle les hommes couraient un risque beaucoup plus élevé que les femmes (9 contre 3) (tableau 4, tableau D en annexe).
Le tableau 6 présente les rapports de risque de mortalité, toutes causes confondues, non corrigés et corrigés comparant les adultes autochtones aux adultes non autochtones. Ces rapports sont élevés, aussi bien chez les hommes que chez les femmes autochtones vivant en milieu urbain (respectivement 1,60 et 2,00). Après avoir évalué différents facteurs comme la taille de la collectivité, la monoparentalité, le degré de scolarité, la catégorie de revenu, le niveau de compétence professionnelle, la situation relative à l'emploi et l'immigration, les rapports de risques ont été ramenés respectivement à 1,22 et 1,68 , ce qui laisse penser que $63 \%$ (pour les hommes) et $32 \%$ (pour les femmes) des écarts constatés dans les rapports de risque peuvent être expliqués par ces variables socioéconomiques.

\section{Analyse}

C'est la première fois qu'une étude en profondeur examine les profils de mortalité d'un grand nombre d'adultes autochtones vivant en milieu urbain au Canada. Il est important de souligner que le lieu de résidence, ainsi que toutes les caractéristiques démographiques et socioéconomiques, ont été vérifiés seulement au début de la période de référence (soit le 4 juin 1991) et qu'elles ne reflètent donc pas nécessairement la réalité tout au long de la période de suivi. Des recherches ont montré en effet que la population autochtone a tendance à déménager plus souvent que les non-Autochtones ${ }^{6}$. Par exemple, environ $70 \%$ des Autochtones (tous âges confondus) vivant dans des régions métropolitaines ont changé de domicile entre 1991 et 1996, 45 \% d'entre eux ayant déménagé au sein de la même collectivité ${ }^{\text {. }}$

Dans la cohorte qui fait l'objet de notre étude, la mortalité des adultes autochtones atteignait des taux plus élevés, l'espérance de vie était plus courte et la probabilité de survie jusqu'à l'âge de 75 ans, inférieure par rapport aux adultes non autochtones vivant dans le même contexte urbain. Ce profil de mortalité plus élevée tend à confirmer les résultats obtenus dans le passé pour les Indiens inscrits vivant à Winnipeg ${ }^{19}$, Vancouver ${ }^{20}$ et dans l'ensemble du Canada ${ }^{14}$. 
TABLEAU 1

Caractéristiques des membres de la cohorte d'Autochtones et de non-Autochtones vivant en milieu urbain selon le lieu de résidence et le sexe, population âgée de 25 ans et plus, à l'exclusion des pensionnaires d'établissements institutionnels, à la date de référence, Canada, 1991

\begin{tabular}{|c|c|c|c|c|c|c|}
\hline & \multicolumn{3}{|c|}{ Autochtones } & \multicolumn{3}{|c|}{ Non-Autochtones } \\
\hline & $\begin{array}{c}\text { Toutes les régions } \\
\text { urbaines }\end{array}$ & $\begin{array}{c}\text { Régions } \\
\text { métropolitaines }^{\mathrm{a}}\end{array}$ & $\begin{array}{c}\text { Petits centres } \\
\text { urbains }^{b}\end{array}$ & $\begin{array}{c}\text { Toutes les régions } \\
\text { urbaines }\end{array}$ & $\begin{array}{c}\text { Régions } \\
\text { métropolitaines }^{\mathrm{a}}\end{array}$ & $\begin{array}{c}\text { Petits centres } \\
\text { urbains }^{\mathrm{b}}\end{array}$ \\
\hline Nombre & 16300 & 10400 & 5900 & 2062700 & 1633600 & 429100 \\
\hline Entre 25 et 44 ans (\%) & 73 & 73 & 73 & 54 & 55 & 53 \\
\hline Parent unique $(\%)$ & 14 & 14 & 14 & 5 & 5 & 5 \\
\hline Sans diplôme d'études secondaires (\%) & 44 & 42 & 46 & 31 & 30 & 36 \\
\hline Diplôme universitaire (\%) & 5 & 6 & 3 & 16 & 17 & 10 \\
\hline En emploi (\%) & 56 & 57 & 55 & 67 & 67 & 64 \\
\hline Nombre & 6900 & 4400 & 2500 & 1013300 & 799800 & 213400 \\
\hline Entre 25 et 44 ans (\%) & 71 & 72 & 71 & 54 & 54 & 52 \\
\hline Âgés de 65 ans et plus (\%) & 5 & 4 & 5 & 14 & 14 & 16 \\
\hline Mariés ou conjoints de fait (\%) & 67 & 64 & 73 & 79 & 78 & 82 \\
\hline Parent unique $(\%)$ & 3 & 3 & 4 & 2 & 2 & 2 \\
\hline Sans diplôme d'études secondaires (\%) & 45 & 43 & 47 & 31 & 29 & 36 \\
\hline Diplôme universitaire (\%) & 5 & 7 & 2 & 18 & 19 & 12 \\
\hline En emploi (\%) & 65 & 66 & 64 & 74 & 75 & 71 \\
\hline Deux quintiles de revenu les plus faibles (\%) & 57 & 56 & 58 & 33 & 34 & 33 \\
\hline Mariées ou conjointes de fait (\%) & 59 & 56 & 63 & 68 & 67 & 70 \\
\hline Parent unique $(\%)$ & 22 & 23 & 22 & 8 & 8 & 8 \\
\hline Sans diplôme d'études secondaires (\%) & 43 & 42 & 45 & 32 & 31 & 36 \\
\hline Diplôme universitaire (\%) & 5 & 6 & 3 & 13 & 15 & 9 \\
\hline En emploi (\%) & 50 & 51 & 49 & 60 & 61 & 57 \\
\hline Deux quintiles de revenu les plus faibles (\%) & 65 & 64 & 65 & 39 & 39 & 39 \\
\hline Limitation d'activité (\%) & 14 & 14 & 14 & 10 & 10 & 11 \\
\hline
\end{tabular}

Source : Étude canadienne de suivi de la mortalité selon le recensement 1991-2001.

a Population $\geq 100000$ habitants

b Population $\geq 10000$ habitants 
TABLEAU 2

Espérance de vie à 25 ans et probabilité de survie jusqu'à l'âge de 75 ans (à la condition de survivre jusqu'à l'âge de 25 ans), pour des adultes autochtones et non autochtones vivant en milieu urbain selon le lieu de résidence et le sexe, population âgée de 25 ans et plus, à l'exclusion des pensionnaires d'établissements institutionnels, à la date de référence, Canada, 1991-2001

\begin{tabular}{|c|c|c|c|c|c|c|}
\hline & Total & IC à $95 \%$ & Hommes & IC à $95 \%$ & Femmes & IC à $95 \%$ \\
\hline \multicolumn{7}{|c|}{ Espérance de vie à l'âge de 25 ans (ans) } \\
\hline \multicolumn{7}{|l|}{ Autochtones } \\
\hline Toutes les régions urbaines & 50,4 & $(49,7-51,1)$ & 48,1 & $(47,1-49,1)$ & 52,7 & $(51,7-53,7)$ \\
\hline Petits centres urbains ${ }^{b}$ & 50,9 & $(49,8-52,0)$ & 48,2 & $(46,6-49,8)$ & 53,6 & $(52,1-55,0)$ \\
\hline \multicolumn{7}{|l|}{ Non-Autochtones } \\
\hline Petits centres urbains ${ }^{\mathrm{b}}$ & 55,5 & $(55,4-55,6)$ & 52,2 & $(52,1-52,4)$ & 58,8 & $(58,7-59,0)$ \\
\hline \multicolumn{7}{|c|}{ Probabilité de survie jusqu'à l'âgé de 75 ans (\%) } \\
\hline \multicolumn{7}{|l|}{ Autochtones } \\
\hline Toutes les régions urbaines & 57,5 & $(54,8-60,1)$ & 52,2 & $(48,2-56,3)$ & 62,7 & $(59,2-66,2)$ \\
\hline Régions métropolitaines ${ }^{\mathrm{a}}$ & 55,4 & $(52,0-58,7)$ & 52,5 & $(47,5-57,6)$ & 58,2 & $(53,6-62,7)$ \\
\hline Petits centres urbains & 70,7 & $(70,3-71,0)$ & 62,9 & $(62,4-63,4)$ & 78,5 & $(78,0-78,9)$ \\
\hline
\end{tabular}

Source : Étude canadienne de suivi de la mortalité selon le recensement 1991-2001

Abréviations : IC, intervalle de confiance.

a Population $\geq 100000$ habitants

b Population $\geq 10000$ habitants

TABLEAU 3

Décès et rapports des taux de mortalité selon le groupe d'âge à la date de référence, le sexe et le lieu de résidence, pour les adultes autochtones et non autochtones vivant en milieu urbain, population âgée de 25 ans et plus, à l'exclusion des pensionnaires d'établissements institutionnels, à la date de référence, Canada, 1991-2001

\begin{tabular}{|c|c|c|c|c|c|c|c|c|c|}
\hline \multirow[b]{2}{*}{$\begin{array}{l}\text { Sexe et ǵroupe d'âge à } \\
\text { la date de référence }\end{array}$} & \multicolumn{3}{|c|}{ Toutes les régions urbaines } & \multicolumn{3}{|c|}{ Régions métropolitaines $^{\mathrm{a}}$} & \multicolumn{3}{|c|}{ Petits centres urbains ${ }^{\mathbf{b}}$} \\
\hline & Décès & RT & IC à $95 \%$ & Décès & RT & IC à 95 \% & Décès & RT & IC à $95 \%$ \\
\hline \multicolumn{10}{|l|}{ Hommes } \\
\hline Total, 25 ans et plus & 563 & 1,56 & $(1,43-1,70)$ & 354 & 1,59 & $(1,43-1,77)$ & 209 & 1,45 & $(1,26-1,66)$ \\
\hline de 25 à 34 ans & 67 & 2,17 & $(1,71-2,77)$ & 42 & 2,14 & $(1,58-2,91)$ & 25 & 2,16 & $(1,45-3,22)$ \\
\hline de 35 à 44 ans & 78 & 1,77 & $(1,41-2,21)$ & 55 & 1,96 & $(1,50-2,56)$ & 23 & 1,41 & $(0,93-2,13)$ \\
\hline de 65 ans à 74 ans & 103 & 1,31 & $(1,08-1,58)$ & 63 & 1,23 & $(0,96-1,58)$ & 40 & 1,41 & $(1,03-1,93)$ \\
\hline 75 ans et plus & 70 & 1,27 & $(1,01-1,61)$ & 35 & 1,27 & $(0,91-1,77)$ & 35 & 1,23 & $(0,89-1,72)$ \\
\hline \multicolumn{10}{|l|}{ Femmes } \\
\hline Total, 25 ans et plus & 563 & 1,94 & $(1,78-2,11)$ & 377 & 2,10 & $(1,89-2,32)$ & 186 & 1,68 & $(1,44-1,97)$ \\
\hline de 25 à 34 ans & 72 & 3,19 & $(2,52-4,04)$ & 52 & 3,71 & $(2,81-4,89)$ & 20 & 2,25 & $(1,43-3,53)$ \\
\hline 75 ans et plus & 71 & 1,13 & $(0,90-1,43)$ & 34 & 0,92 & $(0,66-1,29)$ & 37 & 1,41 & $(1,02-1,95)$ \\
\hline
\end{tabular}

Source : Étude canadienne de suivi de la mortalité selon le recensement 1991-2001.

Note : Rapport des taux normalisés selon l'âge pour l'ensemble des âges

La population de référence (personnes-années à risque) pour la normalisation selon l'âge est extraite de la répartition des Autochtones selon l'âge (groupes d'âge de cinq ans).

Abréviations : IC, intervalle de confiance; RT, rapport des taux.

a Population $\geq 100000$ habitants

${ }^{\mathrm{b}}$ Population $\geq 10000$ habitants 
TABLEAU 4

Décès et taux de mortalité normalisés selon l'âge, par 100000 personnes-années à risque pour des adultes autochtones vivant en milieu urbain, selon le lieu de résidence et le sexe, à l'exclusion des pensionnaires d'établissements institutionnels âgés de 25 ans et plus à la date de référence, Canada, $1991-2001$

\begin{tabular}{|c|c|c|c|c|c|c|c|c|c|}
\hline & \multicolumn{3}{|c|}{ Toutes les régions urbaines } & \multicolumn{3}{|c|}{ Régions métropolitaines ${ }^{a}$} & \multicolumn{3}{|c|}{ Petits centres urbains } \\
\hline & Décès & TMNA & IC à $95 \%$ & Décès & TMNA & IC à $95 \%$ & Décès & TMNA & IC à $95 \%$ \\
\hline \multicolumn{10}{|l|}{ Hommes } \\
\hline Toutes les causes de décès & 563 & 875,4 & $(804,5-952,4)$ & 354 & 880,9 & $(791,0-981,1)$ & 209 & 860,0 & $(749,9-986,3)$ \\
\hline Maladies infectieuses & 22 & 31,8 & $(20,9-48,5)$ & 17 & 39,3 & $(24,2-63,8)$ & 5 & 19,3 & $(8,0-46,3)$ \\
\hline VIH/sida & 15 & 21,3 & $(12,8-35,4)$ & - & - & & - & - & \\
\hline Autres maladies infectieuses & 7 & 10,5 & $(4,9-22,4)$ & - & - & & - & - & \\
\hline Cancer & 132 & 203,8 & $(171,5-242,3)$ & 79 & 197,9 & $(157,7-248,4)$ & 53 & 215,6 & $(164,3-282,9)$ \\
\hline \multicolumn{10}{|l|}{ Cancers de la trachée, des bronches, des } \\
\hline Autres cancers & $\begin{array}{l}51 \\
81\end{array}$ & $\begin{array}{r}79,8 \\
124,1\end{array}$ & $\begin{array}{l}(60,4-105,4) \\
(99,6-154,6)\end{array}$ & $\begin{array}{l}32 \\
47\end{array}$ & $\begin{array}{r}83,5 \\
114,4\end{array}$ & $\begin{array}{l}(58,2-119,8) \\
(85,5-153,1)\end{array}$ & $\begin{array}{l}19 \\
34\end{array}$ & $\begin{array}{r}76,7 \\
138,9\end{array}$ & $\begin{array}{l}(48,7-120,9) \\
(99,0-194,9)\end{array}$ \\
\hline Maladies endocriniennes & 16 & 24,2 & $(14,7-39,7)$ & 7 & 16,5 & $(7,8-34,9)$ & 9 & 36,1 & $(18,7-69,9)$ \\
\hline Appareil circulatoire & 178 & 285,1 & $(245,6-331,0)$ & 107 & 279,5 & $(229,6-340,3)$ & 71 & 299,3 & $(236,5-378,9)$ \\
\hline Cardiopathie ischémique & 116 & 185,0 & $(153,8-222,5)$ & 71 & 189,1 & $(148,6-240,8)$ & 45 & 193,0 & $(143,6-259,4)$ \\
\hline Autres maladies de l'appareil circulatoire & 62 & 100,1 & $(77,7-128,9)$ & 36 & 90,4 & $(64,5-126,7)$ & 26 & 106,3 & $(72,0-157,0)$ \\
\hline Maladies respiratoires & 39 & 67,5 & $(49,1-92,7)$ & 22 & 63,4 & $(41,0-98,0)$ & 17 & 71,0 & $(43,8-115,1)$ \\
\hline Maladies de l'appareil digestif & 37 & 60,2 & $(42,0-86,2)$ & 28 & 73,5 & $(49,5-109,1)$ & 9 & 33,8 & $(17,5-65,3)$ \\
\hline Causes externes & 97 & 138,3 & $(113,3-168,8)$ & 64 & 142,4 & $(111,4-182,0)$ & 33 & 132,9 & $(94,4-187,1)$ \\
\hline Suicide & 23 & 32,3 & $(21,4-48,6)$ & 17 & 37,0 & $(23,0-59,6)$ & 6 & 23,7 & $(10,6-52,8)$ \\
\hline Accident de la route & 23 & 33,2 & $(22,0-50,0)$ & 14 & 31,7 & $(18,8-53,6)$ & 9 & 37,0 & $(19,2-71,4)$ \\
\hline Autres causes externes & 51 & 72,9 & $(55,4-95,9)$ & 33 & 73,7 & $(52,4-103,7)$ & 18 & 72,1 & $(45,4-114,6)$ \\
\hline Toutes les autres causes & 42 & 64,5 & $(47,5-87,6)$ & 30 & 68,5 & $(47,8-98,2)$ & 12 & 51,9 & $(29,3-91,9)$ \\
\hline Maladies liées au tabac & 81 & 130,3 & $(104,4-162,5)$ & 51 & 137,6 & $(103,3-183,1)$ & 30 & 122,4 & $(85,3-175,8)$ \\
\hline Maladies liées à l'alcool & 29 & 41,7 & $(28,8-60,3)$ & 20 & 44,8 & $(28,7-70,0)$ & 9 & 36,0 & $(18,7-69,5)$ \\
\hline Maladies liées aux drogues & 15 & 20,6 & $(12,4-34,2)$ & - & - & & - & - & \\
\hline $\begin{array}{l}\text { Susceptibles d'être évités par intervention } \\
\text { médicale ( }<75 \text { ans) }\end{array}$ & 48 & 68,6 & $(51,7-91,1)$ & 30 & 66,3 & $(46,4-94,9)$ & 18 & 72,7 & $(45,7-115,7)$ \\
\hline \multicolumn{10}{|l|}{ Femmes } \\
\hline Toutes les causes de décès & 563 & 615,9 & $(566,2-670,0)$ & 377 & 657,3 & $(593,0-728,7)$ & 186 & 559,4 & $(478,9-653,5)$ \\
\hline Maladies infectieuses & 20 & 20,9 & $(13,5-32,4)$ & 12 & 20,0 & $(11,4-35,3)$ & 8 & 23,6 & $(11,7-47,2)$ \\
\hline VIH/sida & 6 & 6,1 & $(2,8-13,6)$ & - & - & & - & - & \\
\hline Autres maladies infectieuses & 14 & 14,8 & $(8,7-25,0)$ & - & - & & - & - & \\
\hline Cancer & 153 & 162,9 & $(139,0-190,9)$ & 98 & 166,9 & $(136,8-203,6)$ & 55 & 158,5 & $(121,5-206,8)$ \\
\hline $\begin{array}{l}\text { Cancers de la trachée, des bronches, } \\
\text { des poumons }\end{array}$ & & & & & & & & & \\
\hline $\begin{array}{l}\text { des poumons } \\
\text { Cancer du sein }\end{array}$ & $\begin{array}{l}36 \\
26\end{array}$ & $\begin{array}{l}38,6 \\
27,1\end{array}$ & $\begin{array}{l}(27,9-53,6) \\
(18,4-39,8)\end{array}$ & $\begin{array}{l}26 \\
14\end{array}$ & $\begin{array}{l}45,9 \\
23,1\end{array}$ & $\begin{array}{l}(31,2-67,5) \\
(13,7-39,1)\end{array}$ & $\begin{array}{l}10 \\
12\end{array}$ & $\begin{array}{l}27,3 \\
34,7\end{array}$ & $\begin{array}{l}(14,6-50,9) \\
(19,7-61,2)\end{array}$ \\
\hline Autres cancers & 91 & 97,2 & $(79,1-119,4)$ & 58 & 97,9 & $(75,6-126,7)$ & 33 & 96,5 & $(68,4-136,1)$ \\
\hline Maladies endocriniennes & 23 & 25,2 & $(16,7-37,9)$ & 17 & 28,6 & $(17,7-46,0)$ & 6 & 18,6 & $(8,4-41,6)$ \\
\hline Appareil circulatoire & 154 & 178,4 & $(151,4-210,3)$ & 108 & 197,3 & $(162,3-240,0)$ & 46 & 158,2 & $(111,3-224,9)$ \\
\hline Cardiopathie ischémique & 72 & 83,5 & $(65,7-106,2)$ & 50 & 89,0 & $(67,2-117,8)$ & 22 & 78,9 & $(47,7-130,7)$ \\
\hline Autres maladies de l'appareil circulatoire & 82 & 94,9 & $(75,8-119,0)$ & 58 & 108,3 & $(82,6-142,2)$ & 24 & 79,3 & $(48,6-129,4)$ \\
\hline Maladies respiratoires & 34 & 37,9 & $(26,8-53,5)$ & 23 & 41,5 & $(27,3-62,9)$ & 11 & 30,0 & $(16,6-54,3)$ \\
\hline Maladies de l'appareil digestif & 53 & 55,9 & $(42,7-73,2)$ & 38 & 62,6 & $(45,5-86,1)$ & 15 & 43,5 & $(26,2-72,5)$ \\
\hline Causes externes & 58 & 59,9 & $(46,3-77,5)$ & 43 & 70,5 & $(52,2-95,1)$ & 15 & 42,6 & $(25,7-70,8)$ \\
\hline Suicide & 14 & 14,3 & $(8,5-24,1)$ & - & - & & - & - & \\
\hline Accident de la route & 15 & 15,6 & $(9,4-26,0)$ & 11 & 18,1 & $(10,0-32,6)$ & 4 & 11,4 & $(4,3-30,4)$ \\
\hline Autre cause externe (à l'exclusion du suicide) & 29 & 30,0 & $(20,8-43,1)$ & - & - & & - & - & \\
\hline Autre cause externe (y compris le suicide) & 43 & 44,2 & $(32,8-59,7)$ & 32 & 52,4 & $(37,0-74,1)$ & 11 & 31,2 & $(17,3-56,5)$ \\
\hline Toutes les autres causes & 68 & 74,9 & $(58,8-95,3)$ & 38 & 70,0 & $(50,1-97,8)$ & 30 & 84,3 & $(58,9-120,8)$ \\
\hline Maladies liées au tabac & 54 & 57,8 & $(44,3-75,6)$ & 36 & 62,5 & $(45,0-86,8)$ & 18 & 49,1 & $(30,8-78,0)$ \\
\hline Maladies liées à l'alcool & 33 & 34,2 & $(24,3-48,1)$ & 24 & 39,3 & $(26,4-58,7)$ & 9 & 25,4 & $(13,2-48,9)$ \\
\hline Maladies liées aux drogues & 24 & 24,5 & $(16,4-36,6)$ & 17 & 27,5 & $(17,1-44,3)$ & 7 & 19,6 & $(9,3-41,1)$ \\
\hline $\begin{array}{l}\text { Susceptibles d'être évités par intervention } \\
\text { médicale (< } 75 \text { ans) }\end{array}$ & 86 & 92,1 & $(74,5-113,8)$ & 60 & 101,5 & $(78,8-130,8)$ & 26 & 77,0 & $(52,4-113,4)$ \\
\hline
\end{tabular}

Source : Étude canadienne de suivi de la mortalité selon le recensement 1991-2001.

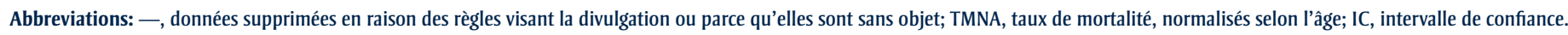

${ }^{\text {a }}$ Population $\geq 100000$ habitants

b Population $\geq 10000$ habitants 
TABLEAU 5

Rapports de taux normalisés selon l'âgée, répartition selon les causes principales de décès et selon le sexe, chez les adultes autochtones et non autochtones vivant en milieu urbain, population âgée de 25 ans et plus, à l'exclusion des pensionnaires d'établissements institutionnels, à la date de référence, Canada, 1991-2001

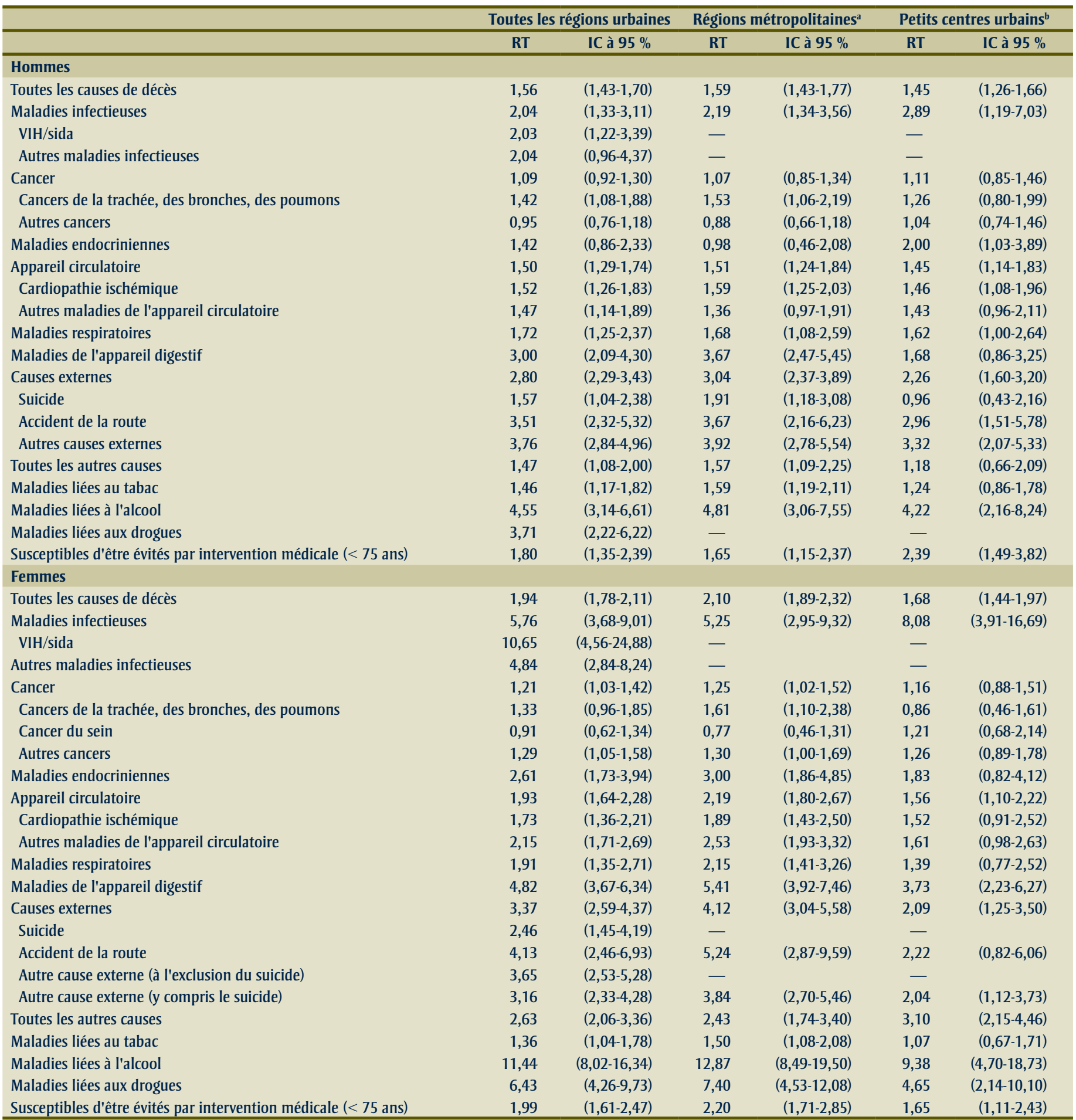

Source : Étude canadienne de suivi de la mortalité selon le recensement 1991-2001.

Abréviations : - , données supprimées en raison des règles visant la divulgation ou parce qu'elles sont sans objet; IC, intervalle de confiance; RT, rapport de taux.

La population de référence (personnes-années à risque) pour la normalisation selon l'âge est extraite de la répartition des Autochtones selon l'âge (groupes d'âge de cinq ans).

a Population $\geq 100000$ habitants

b Population $\geq 10000$ habitants 
TABLEAU 6

Rapports des risques de mortalité corrigés et non corrigés, toutes causes confondues, chez les adultes autochtones et non autochtones résidant dans toutes les régions urbaines, selon le sexe, population âgée de 25 ans et plus, à l'exclusion des pensionnaires des établissements institutionnels, à la date de référence, Canada 1991-2001

\begin{tabular}{|c|c|c|c|c|c|c|c|c|}
\hline \multirow{3}{*}{ Caractéristique à la date de référence } & \multicolumn{4}{|c|}{ Hommes } & \multicolumn{4}{|c|}{ Femmes } \\
\hline & \multicolumn{2}{|c|}{ Avant correction } & \multicolumn{2}{|c|}{ Après correction } & \multicolumn{2}{|c|}{ Avant correction } & \multicolumn{2}{|c|}{ Après correction } \\
\hline & $\begin{array}{c}\text { Rapport } \\
\text { de risque }\end{array}$ & IC à $95 \%$ & $\begin{array}{c}\text { Rapport } \\
\text { de risque }\end{array}$ & IC à 95 \% & $\begin{array}{l}\text { Rapport } \\
\text { de risque }\end{array}$ & IC à $95 \%$ & $\begin{array}{l}\text { Rapport } \\
\text { de risque }\end{array}$ & IC à $95 \%$ \\
\hline \multicolumn{9}{|l|}{ Autochtone } \\
\hline Oui & 1,60 & $(1,47-1,73)$ & 1,22 & $(1,13-1,33)$ & 2,00 & $(1,84-2,17)$ & 1,68 & $(1,55-1,83)$ \\
\hline Non (précision) & 1,00 & $\ldots$ & 1,00 & $\ldots$ & 1,00 & $\ldots$ & 1,00 & $\ldots$ \\
\hline Âge (ans) & 1,10 & $(1,10-1,10)$ & 1,09 & $(1,09-1,09)$ & 1,10 & $(1,10-1,10)$ & 1,09 & $(1,09-1,09)$ \\
\hline \multicolumn{9}{|l|}{ Monoparentalité } \\
\hline Oui & $\ldots$ & $\ldots$ & 1,04 & $(0,99-1,08)$ & $\ldots$ & $\ldots$ & 1,10 & $(1,07-1,13)$ \\
\hline Non (précision) & $\ldots$ & $\ldots$ & 1,00 & $\ldots$ & $\ldots$ & $\ldots$ & 1,00 & $\ldots$ \\
\hline \multicolumn{9}{|l|}{ Lieu de résidence } \\
\hline Régions métropolitaines & $\ldots$ & $\ldots$ & 1,01 & $(1,00-1,03)$ & $\ldots$ & $\ldots$ & 0,99 & $(0,98-1,01)$ \\
\hline Petits centres urbains & $\ldots$ & $\ldots$ & 1,00 & $\ldots$ & $\ldots$ & $\ldots$ & 1,00 & $\ldots$ \\
\hline \multicolumn{9}{|l|}{ Degré de scolarité } \\
\hline Sans diplôme d'études secondaires & $\ldots$ & $\ldots$ & 1,35 & $(1,32-1,39)$ & $\ldots$ & $\ldots$ & 1,24 & $(1,19-1,28)$ \\
\hline Diplôme d'études secondaires & $\ldots$ & $\ldots$ & 1,22 & $(1,19-1,25)$ & $\ldots$ & $\ldots$ & 1,15 & $(1,11-1,19)$ \\
\hline Diplôme d'études postsecondaires & $\ldots$ & $\ldots$ & 1,09 & $(1,06-1,13)$ & $\ldots$ & $\ldots$ & 1,07 & $(1,03-1,11)$ \\
\hline Diplôme universitaire (précision) & $\ldots$ & $\ldots$ & 1,00 & $\ldots$ & $\ldots$ & $\ldots$ & 1,00 & $\ldots$ \\
\hline \multicolumn{9}{|l|}{ Quintile de revenu } \\
\hline Quintile 1 - inférieur & $\ldots$ & $\ldots$ & 1,41 & $(1,38-1,44)$ & $\ldots$ & $\ldots$ & 1,30 & $(1,27-1,33)$ \\
\hline Quintile 2 & $\ldots$ & $\ldots$ & 1,18 & $(1,16-1,20)$ & $\ldots$ & $\ldots$ & 1,13 & $(1,10-1,15)$ \\
\hline Quintile 3 & $\ldots$ & $\ldots$ & 1,10 & $(1,07-1,12)$ & $\ldots$ & $\ldots$ & 1,08 & $(1,05-1,11)$ \\
\hline Quintile 4 & $\ldots$ & $\ldots$ & 1,04 & $(1,01-1,06)$ & $\ldots$ & $\ldots$ & 1,04 & $(1,01-1,07)$ \\
\hline Quintile 5 - supérieur (précision) & $\ldots$ & $\ldots$ & 1,00 & $\ldots$ & $\ldots$ & $\ldots$ & 1,00 & $\ldots$ \\
\hline \multicolumn{9}{|c|}{ Profession - catégories en fonction des compétences } \\
\hline Poste de professionnel (précision) & $\ldots$ & $\ldots$ & 1,00 & $\ldots$ & $\ldots$ & $\ldots$ & 1,00 & $\ldots$ \\
\hline Poste de gestion & $\ldots$ & $\ldots$ & 0,99 & $(0,95-1,03)$ & $\ldots$ & $\ldots$ & 1,07 & $(0,99-1,15)$ \\
\hline Poste spécialisé/technique/de supervision & $\ldots$ & $\ldots$ & 1,09 & $(1,05-1,13)$ & $\ldots$ & $\ldots$ & 1,10 & $(1,04-1,16)$ \\
\hline Poste de spécialisation moyenne & $\ldots$ & $\ldots$ & 1,19 & $(1,14-1,23)$ & $\ldots$ & $\ldots$ & 1,11 & $(1,05-1,17)$ \\
\hline Poste non spécialisé & $\ldots$ & $\ldots$ & 1,27 & $(1,22-1,33)$ & $\ldots$ & $\ldots$ & 1,18 & $(1,11-1,26)$ \\
\hline Sans profession & $\ldots$ & $\ldots$ & 1,29 & $(1,24-1,34)$ & $\ldots$ & $\ldots$ & 1,29 & $(1,22-1,36)$ \\
\hline \multicolumn{9}{|l|}{ Situation relative à l'emploi } \\
\hline Sans emploi (précision) & $\ldots$ & $\ldots$ & 1,00 & $\ldots$ & $\ldots$ & $\ldots$ & 1,00 & $\ldots$ \\
\hline En emploi & $\ldots$ & $\ldots$ & 0,82 & $(0,79-0,85)$ & $\ldots$ & $\ldots$ & 0,89 & $(0,84-0,94)$ \\
\hline Inactif & $\ldots$ & $\ldots$ & 1,16 & $(1,11-1,20)$ & $\ldots$ & $\ldots$ & 1,09 & $(1,03-1,16)$ \\
\hline \multicolumn{9}{|l|}{ Lieu de naissance } \\
\hline Canada (précision) & $\ldots$ & $\ldots$ & 1,00 & $\ldots$ & $\ldots$ & $\ldots$ & 1,00 & $\ldots$ \\
\hline À l'étranger & $\ldots$ & $\ldots$ & 0,74 & $(0,73-0,75)$ & $\ldots$ & $\ldots$ & 0,84 & $(0,83-0,86)$ \\
\hline
\end{tabular}

Source : Étude canadienne de suivi de la mortalité selon le recensement 1991-2001.

Note : ... sans objet 
Les taux de mortalité plus élevés des Autochtones sont liés, pense-t-on, à un large éventail de déterminants sociaux qui exercent leur influence sur la santé sous des formes complexes et dynamiques, depuis la petite enfance jusqu'à la vieillesse ${ }^{34,35}$. Notre étude démontre que les variables socioéconomiques contribuent grandement aux taux de mortalité élevés des adultes autochtones vivant en milieu urbain, particulièrement chez les hommes.

Les résultats ventilés selon les principales causes de décès font ressortir différentes tendances liées aux facteurs de risque. Si on les compare à ceux des membres de la cohorte non autochtones, les rapports de taux atteints par les adultes autochtones vivant en milieu urbain sont particulièrement élevés pour certaines causes de décès comme les maladies de l'appareil digestif, les accidents de la route, les maladies liées à la consommation d'alcool et de drogues et le VIH/sida, tandis que les rapports concernant d'autres causes, notamment toutes les formes de cancer, sont semblables ou légèrement plus élevés. Dans ces cas, les rapports de taux établis pour les adultes autochtones vivant dans des régions métropolitaines et ceux vivant dans de petits centres urbains sont généralement semblables.

Les maladies de l'appareil circulatoire constituent la cause la plus courante de décès tant chez les hommes que les femmes autochtones âgés de 25 ans ou plus, comptant pour respectivement $32 \%$ et $29 \%$ de tous les décès. La plupart de ces décès sont imputables à la cardiopathie ischémique. Les risques relatifs de décès des suites de maladies de l'appareil circulatoire sont élevés chez les adultes autochtones, comme cela a été constaté dans le cas des Indiens inscrits en ColombieBritannique $^{20}$. Une étude effectuée par les Premières nations de l'Ontario a établi que le taux d'hospitalisation résultant d'une cardiopathie ischémique avait considérablement augmenté de 1981 à $1997^{36}$ : certains des participants à cette étude ont peut-être déménagé dans une zone urbaine afin d'obtenir des services de santé spécialisés inacessibles en milieu rural ou dans des régions éloignées.
Les décès dus au cancer équivalent approximativement à un quart de tous les décès chez les adultes autochtones vivant en milieu urbain. Les rapports de taux de mortalité calculés par rapport aux non-Autochtones et concernant toutes les formes de cancer ne sont pas élevés chez les hommes autochtones vivant en milieu urbain et peu élevés chez les femmes autochtones, un résultat semblable à celui obtenu auprès des Indiens inscrits en Colombie-Britannique ${ }^{20}$. Le regroupement de toutes les formes de cancer peut, toutefois, masquer d'importants écarts, car une autre recherche effectuée précédemment a montré que les Autochtones sont davantage susceptibles de souffrir de certaines formes de cancer, mais pas de certaines autres ${ }^{37-41}$. La taille restreinte de l'échantillon dans le cadre de cette étude n’a pas permis de procéder à une analyse détaillée de toutes les formes de cancer, mais nos résultats indiquent que les risques de cancer de la trachée, des bronches et du poumon sont plus élevés parmi les adultes autochtones vivant en milieu urbain, particulièrement chez ceux qui résident dans des régions métropolitaines. La prévalence du tabagisme, un facteur de risque dans le cas du cancer du poumon, mais aussi d'autres cancers, était plus de deux fois plus importante chez les Autochtones âgés de 15 ans ou plus que chez les non-Autochtones (43\% contre $21 \%)^{42}$.

D'autres études ont montré que l'épidémie de VIH/sida est particulièrement virulente au sein des populations autochtones, surtout chez les jeunes ${ }^{20,43}$. Les résultats de notre étude le confirment : les rapports de taux de mortalité due au VIH/sida sont plus de deux fois plus élevés chez les hommes autochtones et ils sont plus de 10 fois plus élevés chez les femmes autochtones. Parmi les Indiens inscrits en ColombieBritannique, le taux des décès causés par le VIH ont plus que doublé entre 1993 et $2006^{20}$.

Le risque de décès causé par des blessures externes, par exemple lors d'un accident de la route ou d'un suicide, était plus élevé chez les adultes autochtones que chez les adultes non autochtones vivant en milieu urbain. D’autres études ont également montré que les Indiens inscrits et les Autochtones en général sont plus susceptibles que les autres Canadiens de mourir à la suite de tels accidents ${ }^{20,44,45}$. On n’a pu procéder à une répartition plus détaillée des différents types de causes externes de décès en raison du nombre relativement faible d'adultes autochtones vivant en milieu urbain et faisant partie de la cohorte, mais le risque de décès provoqué par une cause externe semble plus élevé chez les adultes autochtones vivant dans une région métropolitaine que chez ceux résidant dans de petits centres urbains. Dans cette étude, la proportion de l'ensemble des décès pouvant être attribués à des causes externes est plus faible que dans d'autres études : cette différence s'explique en partie par le fait que notre recherche porte sur une cohorte formée de personnes âgées de 25 ans et plus, alors que les décès survenant en raison de causes externes sont plus courants chez les plus jeunes ${ }^{44,45}$. Notre étude ne tenant pas compte des personnes de moins de 25 ans, il n'a pas été non plus possible de mettre en évidence l'ampleur du problème du suicide : l'âge moyen des personnes décédées par suite d'un suicide est de 27 ans chez les Autochtones du Manitoba comparativement à 45 ans dans le reste de la population manitobaine ${ }^{46}$.

Le risque de décès par suite de maladies liées au tabagisme est supérieur chez les Autochtones de la cohorte vivant en milieu urbain, mais le risque relatif n'est pas aussi important que pour d'autres causes de décès. Comparativement, le risque relatif de décès des suites de maladies liées à la consommation d'alcool est, lui, considérablement plus élevé chez les adultes autochtones (particulièrement les femmes) que chez les adultes non autochtones vivant en milieu urbain. D'autres études ont montré que les Indiens inscrits courent un risque relatif plus grand de mourir des suites de maladies liées à la consommation d'alcool comparativement aux non-Autochtones ${ }^{17,20,47}$. Malgré ce risque relatif accru, les décès survenant chez les adultes autochtones par suite de maladies liées à la consommation d'alcool constituent une proportion plus faible que dans les cas de maladies liées au tabagisme. 
Le nombre de décès survenus avant l'âge de 75 ans et dus à des maladies traitables médicalement est plus élevé chez les adultes autochtones que non autochtones vivant en milieu urbain. Même si la cause de ce risque accru demeure inconnue, une étude effectuée en 2004 a établi que la proportion des personnes déclarant avoir un médecin régulier (médecin de famille) ne variait pas entre les personnes autochtones et non autochtones vivant en milieu urbain au Canada, mais qu'en revanche les Autochtones sont plus susceptibles que les non-Autochtones de déclarer que leurs besoins en matière de soins de santé ne sont pas satisfaits ${ }^{42}$.

\section{Points forts et limites}

L'Étude canadienne de suivi de la mortalité selon le recensement offre une excellente occasion d'examiner à large échelle les profils de mortalité des adultes autochtones vivant en milieu urbain. Pour pouvoir participer à cette étude et pour que le couplage soit réalisable, les répondants devaient avoir complété le questionnaire détaillé du recensement de 1991 et avoir également avoir rempli une déclaration de revenus en 1990 ou 1991. Toute personne n'ayant pas produit de déclaration de revenus (en vertu de l'article 87 de la Loi sur les Indiens, les Indiens inscrits ont droit à une exemption sur les revenus gagnés sur une réserve ou considérés comme tels ${ }^{48}$ ) ou résidant dans un établissement de soins de longue durée, une résidence pour personnes âgées ou une prison ne pouvait faire partie de la cohorte. Malgré cette restriction, nous n'avons pas trouvé de différences importantes dans les caractéristiques démographiques et socioéconomiques entre les répondants au recensement admissibles à l'étude ceux et dont le couplage avec le fichier nominatif a effectivement été réalisé.

À l'âge de 25 ans, les membres de la cohorte avaient une espérance de vie résiduelle plus longue d'un an pour les hommes, et de deux ans pour les femmes par rapport aux résultats des tables de survie pour l'ensemble du Canada 1995-1997.

La détermination des décès était légèrement inférieure chez les Autochtones (entre $95 \%$ et $96 \%$ ) que dans l'ensemble de la cohorte (97 \%). Cet élément entraîne une légère inflexion des taux de mortalité établis pour la population autochtone vivant en milieu urbain, l'ampleur exacte des disparités avec les membres non autochtones de la cohorte pourrait donc être légèrement supérieure à celle qui est indiquée dans cette étude.

Étant donné que le recensement de 1991 ne contenait aucune question concernant l'auto-déclaration d'identité autochtone, notre étude se fonde sur l'ascendance autochtone, le statut d'Indien inscrit ou l'appartenance à une bande indienne ou à une Première nation afin de défnir la population autochtone vivant en milieu urbain. Cette définition a pour conséquence d'exclure bon nombre d'individus qui se seraient auto-identifiés comme Autochtones. En effet, d'après les résultats du recensement de 1996 concernant l'auto-déclaration d'appartenance à un groupe autochtone, environ $8 \%$ des individus s'étant auto-déclarés membres d'un groupe autochtone n'ont pas indiqué d'ascendance autochtone ${ }^{32}$, même si certains d'entre eux étaient des Indiens inscrits ou faisaient partie d'une bande indienne ou d'une des Premières nations.

Certaines études ont fait ressortir des différences relatives aux indicateurs de santé pour les membres des Premières nations, les Inuits et les Métis ${ }^{10}$. Étant donné que cette étude regroupe les Métis, les Inuits et les Premières nations, les différences à l'intérieur de ces groupes n’ont pas pu être déterminées. Les résultats, en outre, ne sont sans doute pas révélateurs du mode de vie des Inuits vivant en milieu urbain, ces derniers ne constituant que $3 \%$ des Autochtones de cette cohorte.

\section{Conclusion}

Jusqu'à maintenant, il y avait peu de données concernant la mortalité au sein de la population autochtone vivant en milieu urbain au Canada. Cette étude nous a permis d'établir que les taux de mortalité des adultes autochtones vivant en milieu urbain étaient plus élevés que ceux des adultes non autochtones vivant dans le même contexte. Les maladies liées à l'appareil circulatoire et les différents types de cancer constituent les deux causes de décès les plus courantes aussi bien chez les adultes autochtones que chez les adultes non autochtones vivant en milieu urbain. Ces risques relatifs étaient particulièrement élevés pour certaines causes de décès, notamment les maladies liées à l'appareil digestif, les accidents de la route, les maladies liées à la consommation d'alcool et de drogues, ainsi que le VIH/sida. En concordance avec d'autres études, nos résultats ont démontré que le statut socioéconomique constituait un facteur important d'explication de ces disparités.

\section{Remerciements}

Le financement de cette étude provient en grande partie de l'Initiative sur la santé de la population canadienne (ISPC) de l'Institut canadien d'information sur la santé (ICIS). Nous voudrions aussi souligner le rôle clé joué par les bureaux provinciaux et territoriaux des statistiques de l'état civil, qui nous ont fourni les données concernant les décès pour la Base canadienne de données sur la mortalité.

Les points de vue exprimés dans cet article n'engagent que les auteurs et ne reflètent pas nécessairement ceux des organismes nommés précédemment ou des institutions auxquels les auteurs sont affiliés.

\section{Références}

1. Kalbach WE. Growth and distribution of Canada's ethnic populations, 1871-1981. Dans : Dreidgder L, dir. Ethnic Canada: identities and inequalities. Toronto (Ont.): Copp Clark Pitman; 1987. p.82-110.

2. Statistique Canada. Peuples autochtones du Canada en 2006 : Inuits, Métis et Premières nations, Recensement de 2006. Ottawa (Ont.), Statistique Canada; 2008. $\mathrm{N}^{\circ}$ 97-558-XIF.

3. Wilson K, Young TK. An overview of Aboriginal health research in the social sciences: current trends and future directions. Int $\mathrm{J}$ Circumpolar Health. 2008;67(2-3):179-89.

4. Young TK. Review of research on aboriginal populations in Canada: relevance to their health needs. BMJ. 2003;327(7412):419-22. 
5. Institute of Urban Studies. First Nations/ Métis/Inuit Mobility Study: final report. Winnipeg (MB): University of Winnipeg; 2004.

6. Norris MJ, Clatworthy S. Mobilité et migration des Autochtones au sein du Canada urbain : résultats, facteurs et conséquences. Dans : Newhouse D, Peters EJ, dirs. Des gens d'ici : les Autochtones en milieu urbain. Ottawa (Ont.), Projet de recherche sur les politiques; 2003. p.51-85.

7. Newhouse D, Peters EJ. Introduction. Dans : Newhouse D, Peters EJ, dirs. Des gens d'ici : les Autochtones en milieu urbain. Ottawa (Ont.): Projet de recherche sur les politiques; 2003. p.5-16.

8. Gracey M, King M. Indigenous health part 1: determinants and disease patterns. Lancet. 2009;374(9683):65-75.

9. Waldram JB, Herring DA, Young TK. Aboriginal health in Canada: historical, cultural, and epidemiological perspectives, $2^{e}$ éd. Toronto (Ont.): University of Toronto Press; 2006.

10. Institut canadien d'information sur la santé. Améliorer la santé des Canadiens. Ottawa (Ont.): Institut canadien d'information sur la santé; 2004.

11. Adelson N. The embodiment of inequity: health disparities in aboriginal Canada. Can J Public Health. 2005;96 Suppl 2:S45-S61.

12. Conseil canadien de la santé. L'état de santé des Premières nations, des Métis et des Inuits du Canada. Toronto (Ont.): Conseil canadien de la santé; 2005.

13. Browne AJ, McDonald H, Elliot D. First Nations urban Aboriginal health research discussion paper: a report for the First Nations Centre, National Aboriginal Health Organization. Ottawa (Ont.): National Aboriginal Health Organization; 2009.

14. Ministère des Affaires indiennes et du Nord Canada. Données ministérielles de base, 2004. Ottawa (Ont.): Ministère des Affaires indiennes et du Nord Canada; 2005. $\mathrm{N}^{\circ} \mathrm{R} 12-7 / 2003 \mathrm{~F}$.
15. Wilkins R, Uppal S, Fines P, Senécal S, Guimond E, Dion R. Espérance de vie dans les régions où vivent les Inuits au Canada, 1989 à 2003. Rapports sur la santé 2008;19(1):1-14.

16. Wilkins R, Tjepkema M, Mustard C, Choinière R. Étude canadienne de suivi de la mortalité selon le recensement, 1991 à 2001. Rapports sur la santé 2008;19(3):27-48.

17. MacMillan HL, MacMillan AB, Offord DR, Dingle JL. Aboriginal health. CMAJ. 1996; 155(11):1569-78.

18. Trovato F. Canadian Indian mortality during the 1980s. Soc Biol. 2000;47(1-2):135-45.

19. Martens PJ, Sanderson D, Jebamani LS. Mortality comparisons of First Nations to all other Manitobans: a provincial population-based look at health inequalities by region and gender. Can J Public Health. 2005;96 Suppl1:S33-8.

20. British Columbia Provincial Health Officer. Pathways to health and healing: $2^{\text {nd }}$ report on the health and well-being of Aboriginal people in British Columbia. Provincial Health Officer's Annual Report 2007. Victoria (BC): Ministry of Healthy Living and Sport; 2009.

21. Fair ME. Generalized record linkage system: Statistics Canada's record linkage software. Austrian Journal of Statistics. 2004; 33(1\&2):37-53.

22. Fair ME, Carpenter M, Aylwin H. Recherche sur la santé : catalogue des projets sur l'hygiène du travail et de l'environnement, 1978 à 2005. Ottawa (Ont.): Statistique Canada; 2006. N 82-581-XIF.

23. Statistique Canada. Couverture. Rapports techniques du recensement. Série des produits de référence. Ottawa (Ont.): Ministère de l'Industrie des Sciences et de la Technologie; 1994. N 92-341F.

24. Statistique Canada. Enquête auprès des peuples autochtones de 1991 : fichier de microdonnées des adultes : guide de l'utilisateur. Ottawa (Ont.): Statistique Canada; 1995.
25. Carriere KC, Roos LL. A method of comparison for standardized rates of lowincidence events. Med Care. 1997;35(1):57-69.

26. Chiang CL. The life table and its applications. Malabar (Fl): Krieger; 1984.

27. Organisation mondiale de la santé. Manuel de la classification statistique internationale des maladies, traumatismes et causes de décès. Neuvième révision. Genève: Organisation mondiale de la santé; 1977.

28. Organisation mondiale de la Santé. Classification statistique internationale des maladies et des problèmes de santé connexes, dixième révision. Genève: Organisation mondiale de la santé; 1992.

29. Mackenbach JP, Stirbu I, Roskam AJ, Schaap MM, Menvielle G, Leinsalu M Kunst AE; European Union Working Group on Socioeconomic Inequalities in Health. Socioeconomic inequalities in health in 22 European countries. N Engl J Med. 2008;358(23):2468-81.

30. Deaths related to drug poisoning: England and Wales, 1999-2003. Health Stat Q. 2005 Spring;(25):52-9.

31. Statistics Canada. Dictionnaire du recensement de 1991. Ottawa (Ont.): Statistique Canada; 1992. N 92-301F.

32. Guimond E. Définitions floues et explosion démographique : identités mouvantes des groupes autochtones au Canada. Dans : Newhouse D, Peters E, dirs. Des gens d'ici : les Autochtones en milieu urbain. Ottawa (Ont.): Projet de recherche sur les politiques; 2003. p.39-54.

33. Puderer HA. Perspectives et mesures de l'urbain [Internet]. Ottawa (Ont.): Statistique Canada; $1^{\text {er }}$ mai 2009 [consultation le 23 novembre 2009]. Consultable en ligne à la page : http://www.statcan.gc.ca/ pub/92f0138m/92f0138m2009001-fra.htm

34. Reading J. Les déterminants sociaux de la santé chez les Autochtones : approche fondée sur le parcours de vie. Ottawa (Ont.): Sous-comité sénatorial sur la santé de la population; 2009. 
35. Reading CL, Wien F. Health inequalities and social determinants of Aboriginal peoples' health. Prince George (BC): National Collaborating Centre for Aboriginal Health; 2009.

36. Shah BR, Hux JE, Zinman B. Increasing rates of ischemic heart disease in the native population of Ontario, Canada. Arch Intern Med. 2000;160(12):1862-66.

37. Band PR, Gallagher RP, Threlfall WJ, Hislop TG, Deschamps M, Smith J. Rate of death from cervical cancer among native Indian women in British Columbia. CMAJ. 1992;147(12):1802-4.

38. Young TK, Kliewer E, Blanchard J, Mayer T. Monitoring disease burden and preventive behavior with data linkage: cervical cancer among aboriginal people in Manitoba, Canada. Am J Public Health. 2000; 90(9):1466-1468.

39. Marrett LD, Chaudhry M. Cancer incidence and mortality in Ontario First Nations, 1968-1991 (Canada). Cancer Causes Control. 2003;14(3):259-68.

40. Tjepkema $M$, Wilkins $R$, Senécal $S$, Guimond E, Penney C. La mortalité chez les Métis et les Indiens inscrits adultes au Canada : étude de suivi sur 11 ans. Rapports sur la santé 2009; 20(4):1-23.

41. Mahoney MC, Michalek AM. A metaanalysis of cancer incidence in United States and Canadian native populations. Int J Epidemiol. 1991;20(2):323-7.

42. Tjepkema M. La santé des Autochtones vivant hors réserve. Rapports sur la santé 2002;13 Suppl:1-18.

43. Agence de la santé publique du Canada. Le point sur la pandémie de $\mathrm{VIH} /$ sida. Ottawa (Ont.): ASPC; 2005.

44. Allard YE, Wilkins R, Berthelot JM. Mortalité prématurée dans les régions sociosanitaires à forte population autochtone. Rapports sur la santé 2004;15(1): 55-66.
45. Santé Canada. Les lésions traumatiques accidentelles et intentionnelles chez les Autochtones du Canada 1990-1999. Ottawa (Ont.): Ministère des Travaux publics et Services des gouvernementaux Canada; 2001. N ${ }^{\circ}$ H35-4/8-1999.

46. Malchy B, Enns MW, Young TK, Cox BJ. Suicide among Manitoba's aboriginal people, 1988 to 1994. CMAJ. 1997;156(8):1133-1138.

47. Mao Y, Moloughney BW, Semenciw RM, Morrison HI. Indian Reserve and registered Indian mortality in Canada. Can J Public Health. 1992;83(5):350-3.

48. Agence du revenu du Canada. Renseignements pour les Indiens inscrits [Internet]. Ottawa (Ont.): Agence du revenu du Canada, 2009 [Consultation le 23 novembre 2009]. Consultable à la page : http:// www.cra-arc.gc.ca/brgnls/stts-fra.html 


\section{Annexe}

TABLEAU A

Répondants ayant rempli le questionnaire détaillé du recensement, membres de la cohorte, taux de couplage du fichier nominal, décès déterminés et personnes-années à risque, population de 25 ans et plus, à l'exclusion des pensionnaires d'établissements institutionnels, à la date de référence, 1991

\begin{tabular}{|c|c|c|c|c|c|}
\hline & Répondants (n) & $\begin{array}{c}\text { Membres de la } \\
\text { cohorte (n) }\end{array}$ & $\begin{array}{c}\text { Taux de couplage } \\
\text { du fichier } \\
\text { nominatif }(\%)\end{array}$ & Décès déterminés (n) & Personnes-années \\
\hline \multicolumn{6}{|c|}{ Autochtones } \\
\hline \multicolumn{6}{|c|}{ Toutes les régions urbaines } \\
\hline Total & 25500 & 16300 & 64 & 1126 & 166570 \\
\hline Hommes & 11300 & 6900 & 61 & 563 & 69580 \\
\hline Femmes & 14200 & 9400 & 66 & 563 & 96990 \\
\hline \multicolumn{6}{|c|}{ Régions métropolitaines ${ }^{\mathrm{a}}$} \\
\hline Total & 15800 & 10400 & 66 & 731 & 106030 \\
\hline Hommes & 7000 & 4400 & 63 & 354 & 44610 \\
\hline Femmes & 8800 & 6000 & 68 & 377 & 61410 \\
\hline \multicolumn{6}{|c|}{ Petits centres urbains ${ }^{b}$} \\
\hline Total & 9700 & 5900 & 61 & 395 & 60540 \\
\hline Hommes & 4300 & 2500 & 58 & 209 & 24970 \\
\hline Femmes & 5400 & 3400 & 64 & 186 & 35570 \\
\hline \multicolumn{6}{|c|}{ Non-Authochtones } \\
\hline \multicolumn{6}{|c|}{ Toutes les régions urbaines } \\
\hline Total & 2644400 & 2062700 & 78 & 192932 & 20844280 \\
\hline Hommes & 1270400 & 1013300 & 80 & 111126 & 10145220 \\
\hline Femmes & 1373900 & 1049400 & 76 & 81806 & 10699060 \\
\hline \multicolumn{6}{|c|}{ Régions métropolitaines ${ }^{\mathrm{a}}$} \\
\hline Total & 2098600 & 1633600 & 78 & 148482 & 16528930 \\
\hline Hommes & 1007700 & 799800 & 79 & 84836 & 8022930 \\
\hline Femmes & 1090900 & 833700 & 76 & 63646 & 8506000 \\
\hline \multicolumn{6}{|c|}{ Petits centres urbains ${ }^{b}$} \\
\hline Total & 545800 & 429100 & 79 & 44450 & 4315350 \\
\hline Hommes & 262700 & 213400 & 81 & 26290 & 2122290 \\
\hline Femmes & 283000 & 215700 & 76 & 18160 & 2193060 \\
\hline
\end{tabular}

Source : Étude canadienne de suivi de la mortalité selon le recensement 1991-2001.

Nota : Les chiffres de population du recensement sont arrondis à la centaine près, ceux des personnes-années sont arrondis

à la dizaine près.

a Population $\geq 100000$ habitants

b Population $\geq 10000$ habitants 
TABLEAU B

Caractéristiques démographiques et socioéconomiques des répondants autochtones faisant partie du champ de l'enquête (admissibles) et des Autochtones membres de la cohorte vivant en milieu urbain, selon le sexe et le lieu de résidence, population âgée de 25 ans et plus, à l'exclusion des pensionnaires d'établissements institutionnels, à la date de référence, 1991

\begin{tabular}{|c|c|c|c|c|c|c|c|c|c|}
\hline & \multicolumn{3}{|c|}{ Toutes les régions urbaines } & \multicolumn{3}{|c|}{ Régions métropolitaines ${ }^{a}$} & \multicolumn{3}{|c|}{ Petits centres urbains ${ }^{b}$} \\
\hline & $\begin{array}{l}\text { Dans le } \\
\text { champ de } \\
\text { l'enquête }\end{array}$ & Cohorte & Rapport & $\begin{array}{l}\text { Dans le champ } \\
\text { de l'enquête }\end{array}$ & Cohorte & Rapport & $\begin{array}{l}\text { Dans le } \\
\text { champ de } \\
\text { l'enquête }\end{array}$ & Cohorte & Rapport \\
\hline & $\%$ & $\%$ & & $\%$ & $\%$ & & $\%$ & $\%$ & \\
\hline \multicolumn{10}{|l|}{ Hommes } \\
\hline Nombre & 49100 & 6900 & & 33100 & 4400 & & 16000 & 2500 & \\
\hline de 25 à 34 & 43 & 42 & 0,97 & 43 & 42 & 0,97 & 42 & 41 & 0,98 \\
\hline de 35 à 44 & 28 & 30 & 1,04 & 28 & 30 & 1,04 & 28 & 29 & 1,05 \\
\hline de 45 à 54 & 16 & 16 & 1,04 & 16 & 16 & 1,03 & 15 & 16 & 1,07 \\
\hline de 55 à 64 & 8 & 8 & 0,95 & 8 & 8 & 0,97 & 9 & 8 & 0,91 \\
\hline de 65 à 74 & 3 & 3 & 1,01 & 3 & 3 & 1,05 & 3 & 3 & 0,93 \\
\hline Conjoint de fait & 19 & 17 & 0,93 & 18 & 16 & 0,90 & 21 & 20 & 0,96 \\
\hline Marié & 42 & 50 & 1,17 & 41 & 48 & 1,17 & 45 & 53 & 1,17 \\
\hline Antérieurement marié & 13 & 10 & 0,79 & 13 & 11 & 0,82 & 13 & 10 & 0,74 \\
\hline \multicolumn{10}{|l|}{ Parent unique } \\
\hline Oui & 4 & 3 & 0,83 & 4 & 3 & 0,79 & 4 & 4 & 0,87 \\
\hline Non & 96 & 97 & 1,01 & 96 & 97 & 1,01 & 96 & 96 & 1,01 \\
\hline \multicolumn{10}{|l|}{ Degré de scolarité } \\
\hline Sans diplôme d'études secondaires & 47 & 45 & 0,94 & 45 & 43 & 0,96 & 51 & 47 & 0,91 \\
\hline Diplôme d'études secondaires & 38 & 40 & 1,05 & 38 & 39 & 1,03 & 38 & 41 & 1,09 \\
\hline \multicolumn{10}{|l|}{ Quintile du revenu } \\
\hline Quintile 1 - inférieur & 39 & 34 & 0,88 & 39 & 34 & 0,89 & 38 & 33 & 0,86 \\
\hline Quintile 2 & 21 & 23 & 1,06 & 21 & 21 & 1,01 & 22 & 25 & 1,16 \\
\hline Quintile 3 & 17 & 19 & 1,12 & 17 & 19 & 1,12 & 16 & 18 & 1,11 \\
\hline Quintile 4 & 14 & 16 & 1,07 & 14 & 16 & 1,10 & 14 & 15 & 1,02 \\
\hline Quintile 5 - supérieur & 9 & 9 & 1,03 & 9 & 9 & 1,07 & 9 & 9 & 0,97 \\
\hline \multicolumn{10}{|l|}{ Limitation d'activité } \\
\hline Non déclarée & 2 & 1 & 0,59 & 2 & 1 & 0,72 & 2 & 1 & 0,39 \\
\hline Non & 82 & 83 & 1,02 & 82 & 83 & 1,01 & 82 & 84 & 1,02 \\
\hline Oui & 17 & 16 & 0,96 & 17 & 16 & 0,96 & 16 & 16 & 0,95 \\
\hline
\end{tabular}


TABLEAU B - SUITE

Caractéristiques démographiques et socioéconomiques des répondants autochtones faisant partie du champ de l'enquête (admissibles) et des Autochtones membres de la cohorte vivant en milieu urbain, selon le sexe et le lieu de résidence, population âgée de 25 ans et plus, à l'exclusion des pensionnaires d'établissements institutionnels, à la date de référence, 1991

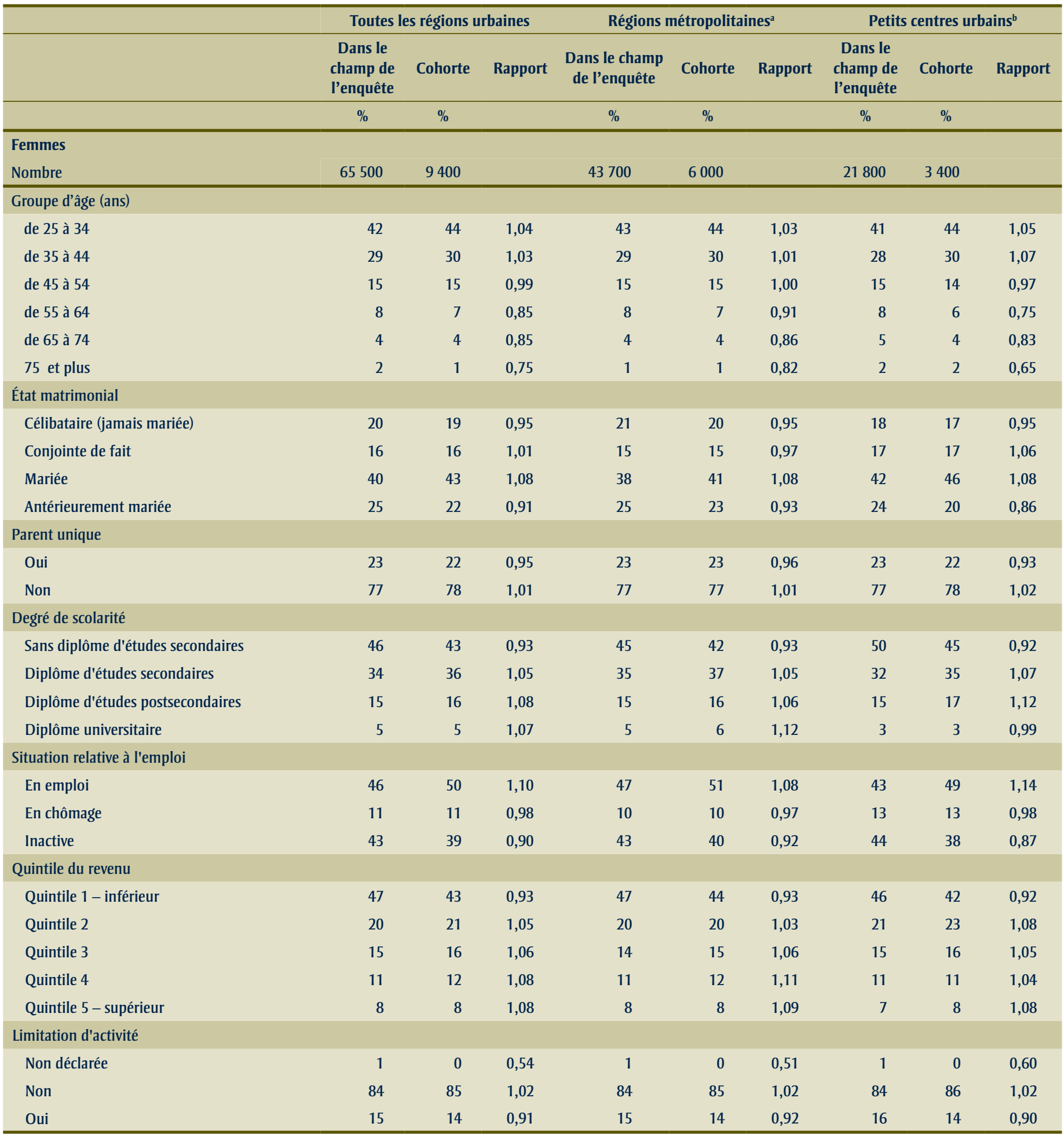

Source : Étude canadienne de suivi de la mortalité selon le recensement 1991-2001.

a Population $\geq 100000$ habitants

${ }^{\mathrm{b}}$ Population $\geq 10000$ habitants 
TABLEAU C

Caractéristiques démographiques et socioéconomiques des répondants non autochtones faisant partie du champ de l'enquête (admissibles) et des non-Autochtones membres de la cohorte vivant en milieu urbain, selon le sexe et le lieu de résidence, population âgée de 25 ans et plus, à l'exclusion des pensionnaires des établissements institutionnels, à la date de référence, 1991

\begin{tabular}{|c|c|c|c|c|c|c|c|c|c|}
\hline & \multicolumn{3}{|c|}{ Toutes les régions urbaines } & \multicolumn{3}{|c|}{ Régions métropolitaines $^{a}$} & \multicolumn{3}{|c|}{ Petits centres urbains ${ }^{b}$} \\
\hline & $\begin{array}{c}\text { Dans le } \\
\text { champ de } \\
\text { l'enquête }\end{array}$ & Cohorte & Rapport & $\begin{array}{l}\text { Dans le champ } \\
\text { de l'enquête }\end{array}$ & Cohorte & Rapport & $\begin{array}{c}\text { Dans le } \\
\text { champ de } \\
\text { l'enquête }\end{array}$ & Cohorte & Rapport \\
\hline \multicolumn{10}{|l|}{ Hommes } \\
\hline Nombre & 6470600 & 1013300 & & 5159500 & 799800 & & 1311100 & 213400 & \\
\hline de 25 à 34 & 29 & 28 & 0,95 & 30 & 28 & 0,94 & 27 & 26 & 0,97 \\
\hline de 35 à 44 & 26 & 26 & 1,02 & 26 & 26 & 1,01 & 26 & 26 & 1,03 \\
\hline de 45 à 54 & 18 & 18 & 1,03 & 18 & 18 & 1,03 & 17 & 18 & 1,02 \\
\hline de 55 à 64 & 14 & 14 & 1,03 & 13 & 14 & 1,04 & 14 & 14 & 1,01 \\
\hline de 65 à 74 & 9 & 10 & 1,04 & 9 & 10 & 1,05 & 11 & 11 & 1,01 \\
\hline \multicolumn{10}{|l|}{ État matrimonial } \\
\hline Conjoint de fait & 7 & 7 & 0,90 & 7 & 6 & 0,90 & 8 & 7 & 0,88 \\
\hline Marié & 67 & 72 & 1,08 & 66 & 72 & 1,08 & 70 & 75 & 1,07 \\
\hline Antérieurement marié & 9 & 7 & 0,81 & 9 & 7 & 0,80 & 9 & 8 & 0,81 \\
\hline \multicolumn{10}{|l|}{ Parent unique } \\
\hline Oui & 2 & 2 & 0,86 & 2 & 2 & 0,86 & 2 & 2 & 0,85 \\
\hline Non & 98 & 98 & 1,00 & 98 & 98 & 1,00 & 98 & 98 & 1,00 \\
\hline \multicolumn{10}{|l|}{ Degré de scolarité } \\
\hline Sans diplôme d'études secondaires & 32 & 31 & 0,97 & 30 & 29 & 0,97 & 38 & 36 & 0,96 \\
\hline Diplôme d'études secondaires & 38 & 38 & 1,01 & 37 & 37 & 1,00 & 40 & 40 & 1,02 \\
\hline \multicolumn{10}{|l|}{ Quintile du revenu } \\
\hline Quintile 1 - inférieur & 16 & 14 & 0,87 & 17 & 14 & 0,87 & 15 & 13 & 0,88 \\
\hline Quintile 2 & 20 & 19 & 0,99 & 20 & 19 & 0,99 & 19 & 19 & 0,99 \\
\hline Quintile 3 & 21 & 21 & 1,02 & 21 & 21 & 1,02 & 21 & 22 & 1,03 \\
\hline Quintile 4 & 21 & 22 & 1,04 & 21 & 22 & 1,04 & 22 & 23 & 1,04 \\
\hline Quintile 5 - supérieur & 22 & 23 & 1,05 & 22 & 23 & 1,05 & 22 & 23 & 1,03 \\
\hline \multicolumn{10}{|l|}{ Limitation d'activité } \\
\hline Non déclarée & 1 & 0 & 0,77 & 1 & 0 & 0,74 & 1 & 1 & 0,88 \\
\hline Non & 89 & 89 & 1,01 & 89 & 90 & 1,01 & 86 & 87 & 1,01 \\
\hline Oui & 11 & 10 & 0,96 & 10 & 10 & 0,97 & 13 & 12 & 0,94 \\
\hline
\end{tabular}


TABLEAU C - SUITE

Caractéristiques démographiques et socioéconomiques des répondants non autochtones faisant partie du champ de l'enquête (admissibles) et des non-Autochtones membres de la cohorte vivant en milieu urbain, selon le sexe et le lieu de résidence, population âgée de 25 ans et plus, à l'exclusion des pensionnaires des établissements institutionnels, à la date de référence, 1991

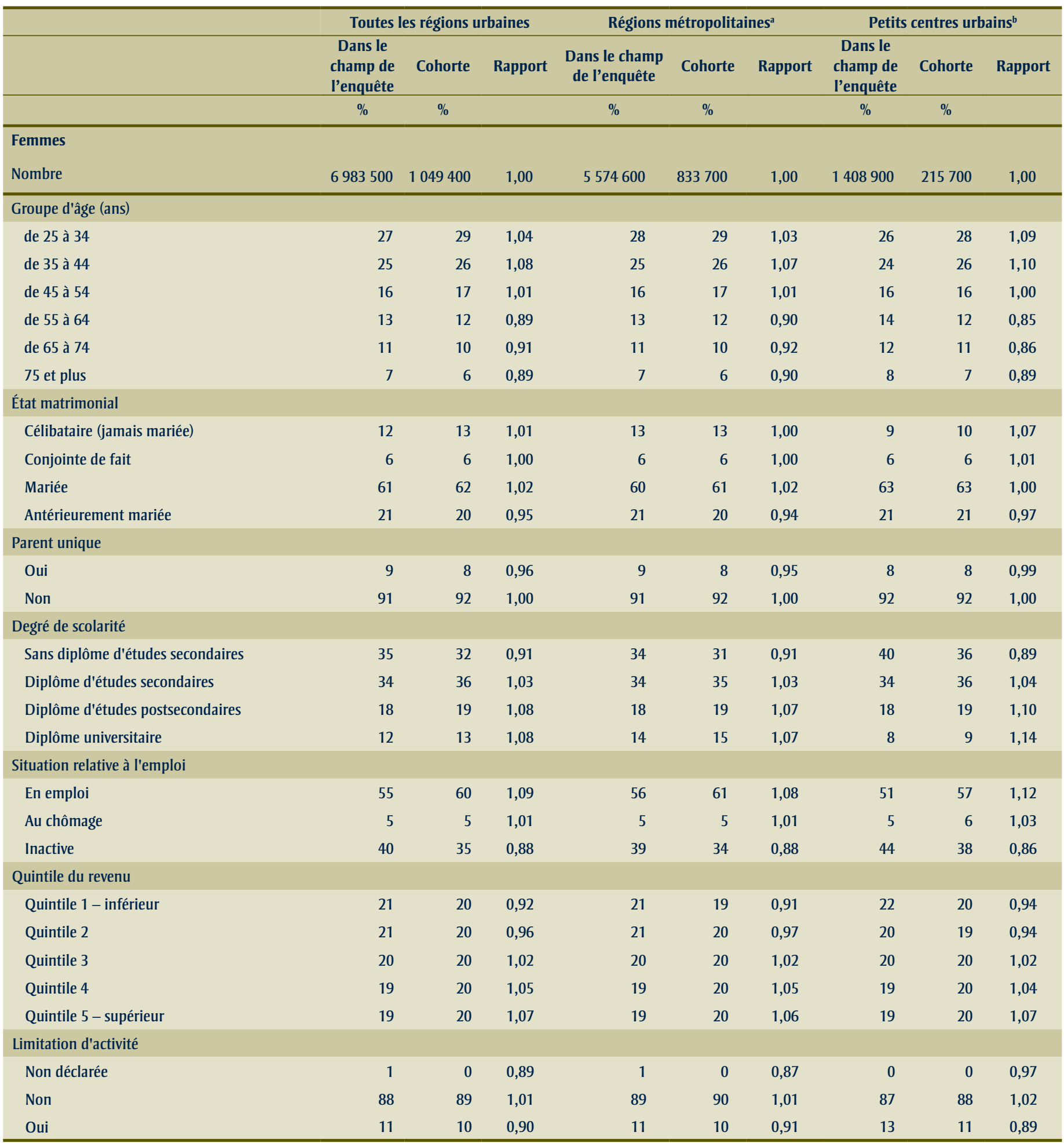

Source : Étude canadienne de suivi de la mortalité selon le recensement 1991-2001.

a Population $\geq 100000$ habitants

b Population $\geq 10000$ habitants 
TABLEAU D

Décès et taux de mortalité normalisés selon l'âge pour 100000 personnes-années à risque pour les adultes non autochtones vivant en milieu urbain selon le lieu de résidence et le sexe, population âgée de $\mathbf{2 5}$ ans et plus, à l'exclusion des pensionnaires d'établissements institutionnels, à la date de référence, Canada, 1991-2001

\begin{tabular}{|c|c|c|c|c|c|c|c|c|c|}
\hline & \multicolumn{3}{|c|}{ Toutes les régions urbaines } & \multicolumn{3}{|c|}{ Régions métropolitaines ${ }^{\mathrm{a}}$} & \multicolumn{3}{|c|}{ Petits centres urbains ${ }^{b}$} \\
\hline & Décès & TMNA & IC à $95 \%$ & Décès & TMNA & IC à $95 \%$ & Décès & TMNA & IC à $95 \%$ \\
\hline \multicolumn{10}{|l|}{ Hommes } \\
\hline Toutes les causes & 111126 & 561,7 & $(558,1-565,4)$ & 84836 & 553,2 & $(549,1-557,3)$ & 26290 & 592,8 & $(584,6-601,0)$ \\
\hline Maladies infectieuses & 1875 & 15,6 & $(14,9-16,4)$ & 1654 & 18,0 & $(17,0-19,0)$ & 221 & 6,7 & $(5,7-7,8)$ \\
\hline VIH/sida & 919 & 10,5 & $(9,8-11,2)$ & 876 & 12,6 & $(11,7-13,5)$ & 43 & 2,4 & $(1,8-3,3)$ \\
\hline Autres maladies infectieuses & 956 & 5,1 & $(4,8-5,5)$ & 778 & 5,4 & $(5,0-5,8)$ & 178 & 4,2 & $(3,6-5,0)$ \\
\hline Cancer & 37073 & 186,7 & $(184,7-188,8)$ & 28544 & 184,8 & $(182,5-187,1)$ & 8529 & 194,1 & $(189,5-198,7)$ \\
\hline $\begin{array}{l}\text { Cancers de la trachée, des bronches, } \\
\text { du poumon }\end{array}$ & 11315 & 56,0 & $(54,9-57,1)$ & 8624 & 54,7 & $(53,5-55,9)$ & 2691 & 60,7 & $(58,3-63,3)$ \\
\hline Autres cancers & 25758 & 130,7 & $(129,0-132,5)$ & 19920 & 130,1 & $(128,1-132,1)$ & 5838 & 133,3 & $(129,6-137,2)$ \\
\hline Maladies endocriniennes & 3463 & 17,1 & $(16,4-17,7)$ & 2647 & 16,8 & $(16,1-17,5)$ & 816 & 18,0 & $(16,7-19,5)$ \\
\hline Appareil circulatoire & 40955 & 190,0 & $(188,0-192,0)$ & 30951 & 185,2 & $(183,0-187,4)$ & 10004 & 207,1 & $(202,6-211,6)$ \\
\hline Cardiopathie ischémique & 25856 & 121,7 & $(120,1-123,3)$ & 19645 & 118,7 & $(117,0-120,5)$ & 6211 & 132,5 & $(128,9-136,2)$ \\
\hline Autres maladies de l'appareil circulatoire & 15099 & 68,3 & $(67,1-69,5)$ & 11306 & 66,5 & $(65,2-67,8)$ & 3793 & 74,6 & $(72,0-77,2)$ \\
\hline Maladies respiratoires & 9390 & 39,2 & $(38,3-40,0)$ & 6971 & 37,8 & $(36,9-38,7)$ & 2419 & 43,8 & $(42,0-45,7)$ \\
\hline Maladies de l'appareil digestif & 3886 & 20,1 & $(19,4-20,8)$ & 3004 & 20,0 & $(19,3-20,8)$ & 882 & 20,2 & $(18,7-21,7)$ \\
\hline Causes externes & 5710 & 49,3 & $(47,9-50,8)$ & 4279 & 46,9 & $(45,3-48,4)$ & 1431 & 58,8 & $(55,5-62,4)$ \\
\hline Suicide & 2063 & 20,5 & $(19,6-21,5)$ & 1547 & 19,4 & $(18,4-20,5)$ & 516 & 24,6 & $(22,4-27,0)$ \\
\hline Accident de la route & 1031 & 9,4 & $(8,8-10,1)$ & 746 & 8,7 & $(8,0-9,4)$ & 285 & 12,5 & $(11,0-14,3)$ \\
\hline Autres causes externes & 2616 & 19,4 & $(18,6-20,3)$ & 1986 & 18,8 & $(17,9-19,8)$ & 630 & 21,7 & $(19,8-23,8)$ \\
\hline Toutes les autres causes & 8774 & 43,8 & $(42,8-44,8)$ & 6786 & 43,7 & $(42,6-44,9)$ & 1988 & 44,1 & $(41,9-46,5)$ \\
\hline Maladies liées au tabac & 18829 & 89,4 & $(88,0-90,8)$ & 14182 & 86,8 & $(85,3-88,3)$ & 4647 & 98,9 & $(95,8-102,0)$ \\
\hline Maladies liées à l'alcool & 1433 & 9,2 & $(8,6-9,7)$ & 1145 & 9,3 & $(8,7-9,9)$ & 288 & 8,5 & $(7,5-9,7)$ \\
\hline Maladies liées aux drogues & 513 & 5,1 & $(5,6-5,1)$ & 410 & 5,6 & $(5,1-6,2)$ & 103 & 5,4 & $(4,4-6,6)$ \\
\hline $\begin{array}{l}\text { Susceptibles d'être évités par } \\
\text { intervention médicale (< } 75 \text { ans) }\end{array}$ & 5540 & 38,1 & $(37,0-39,2)$ & 4473 & 40,1 & $(38,8-41,4)$ & 1067 & 30,4 & $(28,5-32,5)$ \\
\hline
\end{tabular}


TABLEAU D - SUITE

Décès et taux de mortalité normalisés selon l'âge pour 100000 personnes-années à risque pour les adultes non autochtones vivant en milieu urbain selon le lieu de résidence, population âgée de $\mathbf{2 5}$ ans et plus, excluant les pensionnaires d'établissements institutionnels, à la date de référence, Canada, 1991-2001

\begin{tabular}{|c|c|c|c|c|c|c|c|c|c|}
\hline & \multicolumn{3}{|c|}{ Toutes les régions urbaines } & \multicolumn{3}{|c|}{ Réǵions métropolitaines ${ }^{\mathrm{a}}$} & \multicolumn{3}{|c|}{ Petits centres urbains ${ }^{b}$} \\
\hline & Décès & TMNA & IC à $95 \%$ & Décès & TMNA & IC à $95 \%$ & Décès & TMNA & IC à $95 \%$ \\
\hline \multicolumn{10}{|l|}{ Femmes } \\
\hline Toutes les causes & 81806 & 317,6 & $(314,9-320,2)$ & 63646 & 313,6 & $(310,7-316,6)$ & 18160 & 332,5 & $(326,6-338,6)$ \\
\hline Maladies infectieuses & 834 & 3,6 & $(3,3-4,0)$ & 685 & 3,8 & $(3,5-4,2)$ & 149 & 2,9 & $(2,4-3,6)$ \\
\hline VIH/sida & 54 & 0,6 & $(0,4-0,8)$ & 51 & 0,7 & $(0,5-0,9)$ & 3 & 0,2 & $(0,1-0,6)$ \\
\hline Autres maladies infectieuses & 780 & 3,1 & $(2,8-3,3)$ & 634 & 3,1 & $(2,9-3,5)$ & 146 & 2,7 & $(2,2-3,3)$ \\
\hline Cancer & 27256 & 134,3 & $(132,5-136,2)$ & 21495 & 133,6 & $(131,5-135,7)$ & 5761 & 137,2 & $(133,1-141,5)$ \\
\hline $\begin{array}{l}\text { Cancers de la trachée, des bronches, } \\
\text { du poumon }\end{array}$ & 5687 & 29,1 & $(28,2-29,9)$ & 4433 & 28,4 & $(27,5-29,4)$ & 1254 & 31,7 & $(29,8-33,8)$ \\
\hline Cancer du sein & 5158 & 29,7 & $(28,8-30,6)$ & 4111 & 29,9 & $(28,9-31,0)$ & 1047 & 28,8 & $(26,8-30,9)$ \\
\hline Autres cancers & 16411 & 75,5 & $(74,2-76,9)$ & 12951 & 75,2 & $(73,7-76,8)$ & 3460 & 76,7 & $(73,7-79,9)$ \\
\hline Maladies endocriniennes & 2696 & 9,6 & $(9,2-10,1)$ & 2110 & 9,5 & $(9,0-10,0)$ & 586 & 10,2 & $(9,2-11,2)$ \\
\hline Appareil circulatoire & 30369 & 92,4 & $(91,1-93,6)$ & 23298 & 90,0 & $(88,6-91,3)$ & 7071 & 101,4 & $(98,6-104,3)$ \\
\hline Cardiopathie ischémique & 16007 & 48,2 & $(47,3-49,0)$ & 12328 & 47,1 & $(46,2-48,1)$ & 3679 & 52,0 & $(50,1-54,0)$ \\
\hline Autres maladies de l'appareil circulatoire & 14362 & 44,2 & $(43,3-45,1)$ & 10970 & 42,8 & $(41,9-43,8)$ & 3392 & 49,3 & $(47,3-51,4)$ \\
\hline Maladies respiratoires & 6421 & 19,8 & $(19,2-20,4)$ & 4953 & 19,3 & $(18,7-20,0)$ & 1468 & 21,6 & $(20,3-22,9)$ \\
\hline Maladies de l'appareil digestif & 3070 & 11,6 & $(11,1-12,1)$ & 2376 & 11,6 & $(11,0-12,1)$ & 694 & 11,7 & $(10,6-12,8)$ \\
\hline Causes externes & 2995 & 17,8 & $(17,0-18,6)$ & 2273 & 17,1 & $(16,3-18,0)$ & 722 & 20,4 & $(18,6-22,4)$ \\
\hline Suicide & 610 & 5,8 & $(5,3-6,3)$ & 490 & 5,8 & $(5,3-6,4)$ & 120 & 5,8 & $(4,8-7,0)$ \\
\hline Accident de la route & 491 & 3,8 & $(3,4-4,2)$ & 356 & 3,4 & $(3,1-3,9)$ & 135 & 5,1 & $(4,2-6,2)$ \\
\hline Autre cause externe (à l'exclusion du suicide) & 1894 & 8,2 & $(7,7-8,7)$ & 1427 & 7,9 & $(7,3-8,4)$ & 467 & 9,5 & $(8,4-10,7)$ \\
\hline Autre cause externe (y compris le suicide) & 2504 & 14,0 & $(13,3-14,7)$ & 1917 & 13,6 & $(12,9-14,4)$ & 587 & 15,3 & $(13,8-17,0)$ \\
\hline Toutes les autres causes & 8165 & 28,5 & $(27,7-29,2)$ & 6456 & 28,8 & $(27,9-29,7)$ & 1709 & 27,2 & $(25,6-28,9)$ \\
\hline Maladies liées au tabac & 9530 & 42,6 & $(41,6-43,6)$ & 7430 & 41,8 & $(40,7-42,9)$ & 2100 & 45,8 & $(43,6-48,2)$ \\
\hline Maladies liées à l'alcool & 484 & 3,0 & $(2,7-3,3)$ & 382 & 3,1 & $(2,7-3,4)$ & 102 & 2,7 & $(2,2-3,4)$ \\
\hline Maladies liées aux drogues & 413 & 3,8 & $(3,4-4,2)$ & 326 & 3,7 & $(3,3-4,2)$ & 87 & 4,2 & $(3,4-5,3)$ \\
\hline $\begin{array}{l}\text { Susceptibles d'être évités par intervention } \\
\text { médicale ( }<75 \text { ans) }\end{array}$ & 6595 & 46,2 & $(45,0-47,4)$ & 5230 & 46,0 & $(44,7-47,4)$ & 1365 & 46,8 & $(44,2-49,6)$ \\
\hline
\end{tabular}

Source : Étude canadienne de suivi de la mortalité selon le recensement 1991-2001.

Abréviations : - , Données supprimées en raison des règles visant la divulgation ou parce qu'elles sont sans objet; sida, syndrome d'immunodéficience acquise; TMNA, taux de mortalité normalisés selon l'âge; IC, invervalle de confiance; VIH, virus de l'immunodéficience humaine.

La population de référence (personnes-années à risque) pour la normalisation selon l'âge est extraite de la répartition des Autochtones selon l'âge (groupes d'âge de cinq ans)

a Population $\geq 100000$ habitants

b Population $\geq 10000$ habitants 